\title{
Cosmic muon tomography of pure cesium iodide calorimeter crystals
}

\author{
E. Frleža,1, I. Supek ${ }^{\mathrm{b}}$, K. A. Assamagan ${ }^{\mathrm{a}, \mathrm{c}}$, Ch. Brönnimann ${ }^{\mathrm{d}}$, \\ Th. Flügel ${ }^{\mathrm{d}, \mathrm{e}}$, B. Krause ${ }^{\mathrm{d}, \mathrm{f}}$, D. W. Lawrence ${ }^{\mathrm{g}, \mathrm{h}}$, D. Mzavia $^{\mathrm{i}}$,

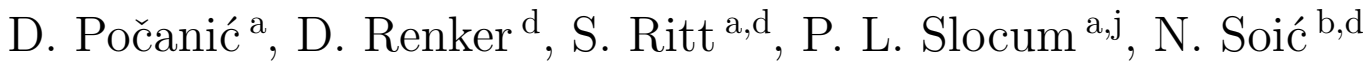 \\ ${ }^{a}$ Department of Physics, University of Virginia, Charlottesville, VA 22901, USA \\ b Institute Rudjer Bošković, Bijenička 46, HR-10000 Zagreb, Croatia \\ ${ }^{\mathrm{c}}$ Physics Department, Hampton University, Hampton, VA 23668, USA \\ ${ }^{d}$ Paul Scherrer Institut, Villigen PSI, CH-5232, Switzerland \\ ${ }^{\mathrm{e}}$ Institut für Teilchenphysik, Eidgenössische Technische Hochschule Zürich, \\ CH-8093 Zürich, Switzerland \\ ${ }^{\mathrm{f}}$ Deutsches Elektronen Synchrotron, D-22603 Hamburg, Germany \\ ${ }^{\mathrm{g}}$ Department of Physics and Astronomy, Arizona State University, Tempe, \\ AZ 85281, USA \\ ${ }^{\mathrm{h}}$ Department of Physics, University of Massachusetts, Amherst, MA 01003, USA \\ ${ }^{\mathrm{i}}$ Institute for High Energy Physics, Tbilisi State University, 380086 Tbilisi, \\ Georgia \\ j Jet Propulsion Laboratory, Pasadena, CA 91109, USA
}

\begin{abstract}
Scintillation properties of pure CsI crystals used in the shower calorimeter being built for precise determination of the $\pi^{+} \rightarrow \pi^{0} e^{+} \nu_{e}$ decay rate are reported. Seventyfour individual crystals, polished and wrapped in Teflon foil, were examined in a multiwire drift chamber system specially designed for transmission cosmic muon tomography. Critical elements of the apparatus and reconstruction algorithms enabling measurement of spatial detector optical nonuniformities are described. Results are compared with a Monte Carlo simulation of the light response of an ideal detector. The deduced optical nonuniformity contributions to the FWHM energy resolution of the PIBETA CsI calorimeter for the $\pi^{+} \rightarrow e^{+} \nu 69.8 \mathrm{MeV}$ positrons and the monoenergetic $70.8 \mathrm{MeV}$ photons were $2.7 \%$ and $3.7 \%$, respectively. The upper limit of optical nonuniformity correction to the $69.8 \mathrm{MeV}$ positron low-energy tail between $5 \mathrm{MeV}$ and $55 \mathrm{MeV}$ was $+0.2 \%$, as opposed to the $+0.3 \%$ tail contribution for the photon of the equivalent total energy. Imposing the $5 \mathrm{MeV}$ calorimeter veto cut to suppress the electromagnetic losses, GEANT-evaluated positron and photon lineshape tail fractions summed over all above-threshold ADCs were found to be $2.36 \pm 0.05$ (stat) \pm 0.20 (sys) \% and $4.68 \pm 0.07$ (stat) \pm 0.20 (sys) \%, respectively.
\end{abstract}


PACS Numbers: 87.59.F; 29.40.Mc; 24.10.Lx

Keywords: Computed tomography; Scintillation detectors; Monte Carlo simulations

$\overline{1}$ Corresponding author; Tel: +1-804-924-6786, fax: +1-804-924-4576, e-mail: frlez@virginia. edu 


\section{Introduction}

The PIBETA collaboration has proposed an experimental program [1] with the aim of making a precise determination of the $\pi^{+} \rightarrow \pi^{0} e^{+} \nu_{e}$ decay rate at the Paul Scherrer Institute (PSI). The proposed technique is designed to achieve an overall level of accuracy in the range of $\sim 0.5 \%$, improving thus on the present branching ratio uncertainty of $4 \%$ [2]. The $(1.025 \pm 0.034) \times 10^{-8}$ pion beta decay $(\pi \beta)$ branching ratio will be remeasured relative to the $10^{4}$ times more probable $\pi^{+} \rightarrow e^{+} \nu_{e}$ decay rate, that is known with the combined statistical and systematic uncertainty of $\sim 0.40 \%[3,4]$.

The Standard Model description of the $\pi \beta$ decay and its radiative corrections [5] enables a stringent test of the conserved vector current (CVC) hypothesis and the unitarity of the Cabibbo-Kobayashi-Maskawa (CKM) quark mixing matrix. The most accurate extraction of the CKM matrix element $V_{u d}$, based on the superallowed Fermi transitions in nuclei, involves the theoretical nuclear overlap corrections that dominate the $0.3 \%$ total uncertainty [6]. Recent measurements of nine different nuclear transition rates confirm the CVC hypothesis at the level of $4 \times 10^{-4}$, but violate the three-generation CKM unitarity condition by more than twice the estimated error [7]. Complementary neutron decay experiments yield a result that also differs from unitarity by over two standard deviations, but in the opposite sense [7]. It is of fundamental interest to measure the branching ratio of the $\pi^{+} \rightarrow \pi^{0} e^{+} \nu_{e}$ decay at the $0.5 \%$ level or better to check the consistency with the nuclear and neutron $\beta$ decay values and the unitary prediction of the Minimal Standard Model (MSM). Moreover, the precisely measured $\pi \beta$ branching ratio could be used to constrain masses and couplings of additional neutral gauge bosons in grand unified theories. If such couplings are directly discovered at future collider experiments, precision low-energy data will be an essential ingredient in extending the MSM framework [8].

The central part of the PIBETA detector system is a high-resolution, highly segmented fast shower calorimeter surrounding the active stopping target in a near-spherical geometry. The requirements imposed on the calorimeter design were:

- high energy resolution for effective suppression of background processes;

- high segmentation and fast timing response to handle the high event rates;

- acceptable radiation, mechanical and chemical resistance;

- compact geometry to simplify the operation and reduce the cost.

These design constraints are met in a spherical detector with individual calorimeter modules made from undoped cesium iodide (CsI) crystals. The nonactivated alkali iodides have been known for more than thirty years to exhibit a 
near-ultraviolet emission component when excited by ionizing radiation $[9,10]$. The pure CsI material was reintroduced as a fast, rugged, and relatively inexpensive scintillator material by Kobayashi et al. [11] and Kubota et at. [12,13]. The scintillation characteristics of pure CsI crystals have been reported subsequently by a number of experimental groups. These investigations have covered light readout techniques, scintillation decay times, origins of different emission components, crystal light yields, and energy and timing resolutions [14-19], radiation resistances [20,21], wrapping and tuning methods and the uniformity of light responses $[22,23]$. The design and performance of pure CsI electromagnetic calorimeters have been recently described in Refs. [24] (NMS at LAMPF/BNL), [25,26] (KTeV at Fermilab), and [27] (PHENIX at RHIC).

The PIBETA calorimeter geometry shown in Fig. 1 is obtained by the class II geodesic triangulation of an icosahedron [28]. Selected geodesic breakdown results in 220 truncated hexagonal, pentagonal and trapezial pyramids covering the total solid angle of $0.77 \times 4 \pi$ sr. Additional 20 crystals cover two detector hemispheres open to the beam and act as shower leakage vetoes. The inner radius of the crystal ball is $26 \mathrm{~cm}$, and the axial module length is $22 \mathrm{~cm}$, corresponding to $12 \mathrm{CsI}$ radiation lengths $\left(X_{0}=1.85 \mathrm{~cm}[29]\right)$. There are nine different module shapes: four irregular hexagonal truncated pyramids (we label them HEX-A, HEX-B, HEX-C, and HEX-D), one regular pentagonal (PENT) and two irregular half-hexagonal truncated pyramids (HEX-D1 and HEX-D2), and two trapezohedrons which function as vetoes (VET-1, VET-2). The volumes of our CsI crystal detector modules vary from $797 \mathrm{~cm}^{3}$ (HEX-D1/2) to $1718 \mathrm{~cm}^{3}$ (HEX-C). Dimensioned drawings of the HEX-A and HEX-D1 crystal shapes are shown as examples in two panels of Fig. 2.

All key components of the complete PIBETA detector have been prototyped and built and have met the required specifications [30]. All the detector components were delivered to PSI by mid-1998. The final assembly of the apparatus was followed by the in-beam commissioning and calibration. "Production" measurements are scheduled for 1999, and will extend over two more years to complete the first phase of the project, a $\sim 0.5 \%$ measurement of the $\pi^{+} \rightarrow \pi^{0} e^{+} \nu$ decay rate.

The quality of the delivered CsI calorimeter crystals was controlled in the following program of measurements:

(1) check precisely the crystal physical dimensions against the specifications;

(2) study the effects of different crystal surface treatments and wrapping configurations on the energy and timing resolution and on the uniformity of light response;

(3) determine the percentage of the fast scintillation component in the light output;

(4) measure the contribution of detector photoelectron statistics to energy 
resolution;

(5) find the temperature dependence of a detector's ADC readings;

(6) determine axial and transverse optical nonuniformities for each crystal.

The ultimate goal of the program was to provide the input parameters for a Monte Carlo simulation of the calorimeter response and to deduce the effects of CsI crystal light yields and optical nonuniformities on the energy lineshapes of detected photons and positrons ranging in energy between $10 \mathrm{MeV}$ and $120 \mathrm{MeV}$.

The spectra generated by cosmic muons have been used to examine the uniformity of light response of two large $(24 \times 36 \mathrm{~cm})$ cylindrical $\mathrm{NaI}(\mathrm{Tl})$ calorimeter crystals in the earlier work of Dowell et al. [31]. Their apparatus relied on plastic scintillator hodoscopes with $3.8 \times 3.8 \mathrm{~cm}^{2}$ cross section for the charged particle tracking. The precision of the trajectory reconstruction was therefore limited to $\sim 4 \mathrm{~cm}$. Such positional resolution was deemed inadequate for our application because the PIBETA CsI crystals have an axial length of $22 \mathrm{~cm}$ with front (back) surface side lengths of less than $4 \mathrm{~cm}(7 \mathrm{~cm})$.

Most of our measurements were done with the tomography apparatus built around three pairs of multiwire drift chambers (MWDC) using cosmic muons as the probe. One dozen PIBETA CsI detectors were examined in a $350 \mathrm{MeV} / \mathrm{c}$ minimum ionizing muon beam of the PSI $\pi$ E1 area, as well as in the $405 \mathrm{MeV} / \mathrm{c}$ penetrating pion and stopping proton beams in the $\pi \mathrm{M} 1$ channel. Selected CsI crystals were also scanned with a $662 \mathrm{keV}{ }^{137} \mathrm{Cs} \gamma$-source. The light yield nonuniformities measured in the beam and with a radioactive-source scan method were compared with more precise cosmic muon tomography data. The experimental apparatus used for a $\gamma$-source crystal scans and the subsequent data reduction were simple enough to be easily adopted by the crystal manufacturers, thus accelerating the subsequent quality control cycle.

\section{Crystal Production, Mechanical Quality Control and Surface Treatment}

Pure CsI crystals produced for the PIBETA calorimeter came from two different sources. Twenty-five crystals were grown and cut to specified shapes in the Bicron Corporation facility in Newbury, Ohio. The rest of the scintillators were grown in the Institute for Single Crystals in Kharkov (AMCRYS), Ukraine. Preliminary examination of fifty-five AMCRYS CsI crystals, including the mechanical and physical tests, was done in the High Energy Institute of Tbilisi University, Georgia.

Manufacturing tolerances of the crystals were specified for the linear dimen- 
sions $(+150 \mu \mathrm{m} /-50 \mu \mathrm{m})$ and for the angular deviations $\left(+0.040^{\circ} /-0.013^{\circ}\right)$. Geometrical dimensions of the machined crystals were measured upon delivery at PSI using the computer-controlled distance-measuring device WENZEL Precision. The machine was programmed to automatically probe the surfaces of a subject crystal with a predefined shape. Each crystal surface plane was scanned with a touch head at six points and the equations of planes were found through these surveyed points. Body vertices, measured with an absolute precision of $2 \mu \mathrm{m}$ and reproducible within $20 \mu \mathrm{m}$, were then compared with the expected theoretical values. Those crystals that failed the imposed geometrical tolerances were returned to the manufacturer for reuse as raw crystal growing material.

After physical measurements crystal surfaces were polished with a mixture of $0.2 \mu \mathrm{m}$ aluminum oxide powder and etylenglycol. Next came a measurement of the light output of the fast CsI scintillation component $(\mathrm{F})$ that is completely decaying in the first $100 \mathrm{~ns}$, relative to the total CsI signal (T), integrated in a $1 \mu \mathrm{s}$ ADC gate. These measurements were made with unwrapped crystals using air-gap coupled photomultiplier tubes (PMTs) and a Tektronix TDS 744 digital oscilloscope. Only the crystals with a fast-to-total component ratio $(\mathrm{F} / \mathrm{T})$ better than 0.7 were accepted. The mean value of fast-to-total ratio for all accepted CsI crystals was 0.788, as shown in Fig. 3.

EMI photomultiplier tubes 9821QKB [32] with $75 \mathrm{~mm}$ diameter cathode were glued to the back faces of hexagonal and pentagonal CsI crystals using a $300 \mu \mathrm{m}$ layer of silicone Sylgard 184 elastomer (Dow Corning RTV silicon rubber plus catalyst). Smaller half-hexagonal and trapezial detector modules were equipped with two inch EMI 9211QKA phototubes [32]. The resulting crystal-photomultiplier tube couplings were strong and permanent, but could be broken by application of a substantial tangential force. The PMT quartz window transparency, peaking at $\sim 380 \mathrm{~nm}$ [32], is approximately matched to the spectral excitation of a pure CsI fast scintillation light component with a maximum room temperature emission at $\sim 310 \mathrm{~nm}$ [15]. The PMT high voltage dividers were modified EMI-recommended bases designed and built at the University of Virginia. These dividers minimized the so-called "super"linearity exhibited by many PMTs well below the onset of saturation. The maximum PMT nonlinearity measured with a pair of light-emitting diodes was less than $2 \%$ over the full dynamic range expected in the $\pi \beta$ decay rate measurement [33].

Four different wrapping materials for lateral crystal surfaces were investigated in wrapping and tuning studies with $405 \mathrm{MeV} / \mathrm{c}$ pion and proton beams in PSI $\mu \mathrm{M} 1$ area:

(1) one to five layers of a $38 \mu \mathrm{m}$ PTFE Teflon sheet $\left(\mathrm{CF}_{2}\right.$ monomer, $\left.X_{0}=16.0 \mathrm{~cm}\right)$;

(2) one to five layers of $30 \mu \mathrm{m}$ Mylar sheet $\left(\mathrm{C}_{5} \mathrm{H}_{4} \mathrm{O}_{2}, X_{0}=28.7 \mathrm{~cm}\right)$; 
(3) a $250 \mu \mathrm{m}$ thick Tyvek fleece (polyethylene $\mathrm{CH}_{2}, X_{0}=131.3 \mathrm{~cm}$ );

(4) a $110 \mu \mathrm{m}$ thick Millipore filter (polyvinylidene fluoride PVDP, $X_{0}=19.0 \mathrm{~cm}$ ) with $0.22 \mu \mathrm{m}$ pores;

(5) a wavelength shifter lacquer treatment of crystal surfaces.

In all cases a primary diffuse reflector was protected by an additional $20 \mu \mathrm{m}$ thick aluminized Mylar cover. These preliminary tests showed more uniform axial light collection from the crystal front section when a polished front crystal surface was covered with a black paper sheet. A similar tuning method, using black paper strips at the front section of $5 \times 6 \times 36 \mathrm{~cm}^{3} \mathrm{CsI}(\mathrm{Tl})$ crystals in order to improve the uniformity of light collection, was recently described in Ref. [23].

The maximum fast-to-total ratio was always achieved with unwrapped crystals because all applied wrapping materials absorb more ultraviolet light which dominates the fast component, than visible light. A two-layered Teflon cover was found to be superior in delivering $\geq 20 \%$ more fast scintillation light component than the other reflectors. It was also comparatively better in not degrading the $\mathrm{F} / \mathrm{T}$ ratio by more than $\sim 2 \%$. These differences in measured light output were reproducible in the beam tests with $\sim 1 \%$ event statistics and estimated $2 \%$ systematic uncertainty. They are subsequently confirmed in more controlled cosmic muon tomography measurements.

On the basis of these findings the adopted standard wrapping configuration for all studied CsI crystals consisted of: (a) the lateral surfaces being wrapped with two layers of Teflon foil plus one layer of aluminized Mylar sheet, and (b) the front crystal surface covered with the black paper template. The back crystal surface with the glued phototube was left uncovered.

Light yield measurements were repeated for a subset of crystals after a six month period to confirm that no appreciable changes occurred as a result of degradation of the surface reflectivities and wrapper material qualities. The measured light output was typically within $5 \%$ of the originally measured value.

The most successful surface treatment of CsI crystals, however, involved painting the lateral crystal surfaces with a waveshift lacquer. We recently studied this method on a large sample of CsI detectors. The light response and uniformity properties were noticeably improved, resulting in $\sim 20 \%$ better energy resolutions for the $70 \mathrm{MeV}$ monoenergetic $e^{ \pm}$and 50-82 MeV tagged photons. The possible degradation and change in the crystal surface reflectivities that could be difficult to account for in the multi-year long precision experiment like the PIBETA is also expected to be arrested by such a treatment. These measurements will be covered in detail in a forthcoming paper [34]. 


\section{Tomographic Apparatus}

A simplified sketch of the tomographic apparatus layout is illustrated in a GEANT rendering showing a few simulated cosmic muon trajectories on Fig. 4. Three identical delay-line readout drift chambers were used to define the cosmic muon tracks intersecting CsI crystals. The chambers were built at the Los Alamos Meson Physics Facility (LAMPF) by the M. Sadler group from the Abilene Christian University. This type of drift chambers has been used reliably for years with several LAMPF spectrometers [35-38]. Each chamber consists of two signal and three ground planes with the nominal active area of $60 \times 60 \mathrm{~cm}^{2}$. The horizontally oriented " $x$ " and " $y$ " signal planes are two orthogonal sets of alternating cathode and anode wires evenly spaced at $0.4064 \mathrm{~cm}$.

The light-tight aluminum box had two drift chamber pairs mounted on its top plate, and one pair fixed below the bottom side. Distances between the centers of chamber pairs 1-3 and 2-3 were $24 \mathrm{~cm}$ and $27 \mathrm{~cm}$, respectively. The dark box could accommodate up to six CsI calorimeter modules at one time. It had feedthrough connectors for twelve signal and twelve high voltage cables as well as six temperature sensor lines and six LED calibration signal cables. A pair of $1 \mathrm{~cm}$ thick plastic scintillators were placed directly below the apparatus and separated from the chamber system by a $5 \mathrm{~cm}$ layer of lead bricks shielding out the soft cosmic ray component. The Monte Carlo simulation (see Sec. 8.1)showed that hard cosmic muons penetrating the frames of the apparatus, CsI crystals and shielding material and triggering two scintillators had a smooth energy threshold starting at $\sim 120 \mathrm{MeV}$. The zenithal angle range of accepted cosmic muon trajectories intersecting all three MWDCs and at least one CsI detector was $\pm 45^{\circ}$.

The chambers were operated with anode wires held at positive voltage between 2400-2600 V. The chamber cathode wires were grounded. The gas mixture was $65 \%$ argon, $35 \%$ isobutane plus $0.1 \%$ isopropyl alcohol. Detection efficiencies of individual chambers for penetrating cosmic muons exceeded $90 \%$, with the combined six-chamber efficiency routinely surpassing $50 \%$.

Anode wires were attached directly to a fast $2.5 \mathrm{~ns} / \mathrm{cm}$ delay-line. Signals from both ends of the delay line were amplified twenty-fold, discriminated at a threshold of $10 \mathrm{mV}$ and connected to two channels of a time-to-digital converter (LCR 2229A TDC). The time difference in the two TDC readings identified the fired anode wire. Cathode lines defining the alternate field $[39,40]$ were connected to one "odd" $(\mathrm{O})$ and one "even" (E) line, and the signals on these lines were processed by a custom-made electronics unit which added and subtracted the analog pulses [41]. The electronic sum of cathode pulses $(\mathrm{O}+\mathrm{E})$ was discriminated and the resulting delayed signal determined the drift 
position timing, and was used to stop another TDC channel. The difference of the cathode signals $(\mathrm{O}-\mathrm{E})$ was digitized with an analog-to-digital converter (LCR 2249A ADC). That information was used to discriminate between the events that produced the ionization tracks left and right of the given anode wire. The $100 \mathrm{~ns}$ integration gate timing was defined by the $(\mathrm{O}+\mathrm{E})$ logic pulse timing. Four measurements were therefore required to find an intersection between a cosmic muon track and one drift chamber plane, namely three TDC values and one ADC value. The schematic diagram of the electronic logic is shown in Fig. 5.

Advantages of the system were good charged particle track resolution $(\sim$ $0.5 \mathrm{~mm}$ root-mean-square in the horizontal plane, Fig. 6), stability to fluctuations in the outside environmental parameters (humidity, temperature and pressure) and low cost of the associated electronics logic and readout. Limited counting rate and restriction to single hit events in the chambers did not represent a drawback in this tomographic application. The apparatus was operated in an air-conditioned room with a controlled humidity level kept below $30 \%$. The temperature at six points inside the dark box as well as absolute time were recorded for every triggered event. The temperature range recorded inside the dark box during three years of data taking was $\left(22^{\circ} \pm 3^{\circ}\right) \mathrm{C}$. The typical temperature gradient was $0.4^{\circ} \mathrm{C} /$ day, leading to average absolute temperature variation of $0.2^{\circ} \mathrm{C}$ and average CsI ADC gain drift of $\sim 0.4 \%$ in a single data taking run (fixed at 250000 triggers, $\sim 6$ hours). Measured light output variations of the CsI crystals and the PMT gain drifts caused by daily temperature cycles were compensated for in the replay analysis (see Sec. 6).

\section{Data Acquisition System}

The computer code used for data acquisition was HIX (Heterogeneous Information Exchange), a data acquisition system developed by S. Ritt at PSI, originally intended for use in small and medium-size nuclear physics experiments [42].

The "frontend" 486 personal computer was connected to a CAMAC crate via a HYTEC 1331 interface that read ADC, TDC, scalar, and temperature sensor units. The $\mathrm{C}$ program running under MS-DOS accessed data and sent it over the network to a VAX 3100 server. A simple communication program on a VAXstation received data from the frontend computer and stored it in a global section buffer. Buffered data were passed by the Logger application to a user-written Analyzer program. Analyzed events were subject to predefined cuts, filled in predefined histograms, and stored in a raw data stream directed simultaneously to a hard disk drive and an 8 -mm tape system. The exper- 
iment was controlled from a PC computer by a Microsoft Windows control program. A windows-based graphical user interface to the program allowed starting, pausing and stopping data acquisition, as well as online inspection of individual raw data words, calculated data words, scalers, assorted efficiencies, one and two-dimensional histograms, and gate, box and Boolean tests. Different PC computers on the Ethernet could make a connection to the VAX server called Link and access the same information remotely.

During data acquisition only those events for which at least two $x$ and two $y$ drift chambers had nonzero ADC and TDC values were written to an diskresident ASCII Data Summary File (DSF). One good event contained 55 raw data words specifying the response of the MWDCs and CsI detectors, instantaneous temperatures and absolute times. That was a full set of observables for which a cosmic muon trajectory could be unambiguously reconstructed. The raw trigger rate was $\sim 13 \mathrm{~Hz}$ with six CsI crystals in the dark box. The average DSF event rate was $\sim 4 \mathrm{~Hz}$. Individual runs were stopped and restarted automatically after 250,000 collected triggers. A total of about $10^{5}$ DSF events per crystal were typically collected in one week of data acquisition. Tomography data for one set of six crystals were usually collected over a two week period.

\section{Drift Chamber Calibration}

Two different trigger configurations were used interchangeably during the data collection. Drift chamber calibration was done with a trigger requiring a twoscintillator coincidence and good ADC and TDC data for at least one drift chamber. In the tomography data acquisition mode, the triple coincidence between two tag scintillators and one CsI detector was required, accompanied by at least one good pair of $x$ and $y$ chamber hits. The rare accidental coincidences involving signals in more than one CsI detector were eliminated from the DSF records in the offline analysis.

The hit anode wire number $n_{A}$ was calculated from

$$
n_{A}=\frac{\left(T_{1}-T_{2}+N \cdot D\right)}{2 D}
$$

where $T_{1}$ and $T_{2}$ were the TDC values for two ends of the anode delay line, $N$ was the number of wires in a chamber (between 71 and 73), and $D$ was the $\sim 2.05 \mathrm{~ns}$ time delay between the adjacent wires. Truncated wire position $x_{T}$ was defined as the nearest integer multiple $n_{A}$ of the $0.8128 \mathrm{~cm}$ wire separation $g$ :

$$
x_{T}=\operatorname{NINT}\left(n_{A} \cdot g\right) .
$$


The anode delay time difference $\left(T_{1}-T_{2}\right)$ depended nonlinearly on the anode position. Linearized anode wire positions for each chamber were approximated by a polynomial expansion

$$
x_{A}=\sum_{i=0}^{m} c_{i} \cdot\left(T_{1}-T_{2}\right)^{i} \text {, }
$$

where the order $m$ of the fit with $\chi^{2}$ per degree of freedom $\chi^{2} /(m-1) \approx 1$ depended on the particular delay line. For our chambers the orders of the fits were 3,4 , or 5 . The coefficients $c_{i}$ were optimized with the program MINUIT [43] that minimized the sum of squared deviations $\left(x_{T}-x_{A}\right)^{2}$ for each chamber in every run.

The drift distance $d_{x}$ was determined from the drift timing $T_{x}$ using the calibration lookup table $f\left(T_{x}\right)$

$$
d_{x}=f\left(T_{x}\right) \cdot T_{x}+\Delta T_{x}
$$

where $\Delta T_{x}$ was the drift time offset. The lookup table was calculated assuming that incoming cosmic muons are distributed uniformly over the equidistantly spaced wires.

The final hit position $x$ was given by expression:

$$
x=x_{T}+(-1)^{n} \cdot \operatorname{sign}\left(\mathrm{ADC}_{\mathrm{O}-\mathrm{E}}+\mathrm{ADC}_{0}\right) \cdot d_{x}+x_{\mathrm{OFF}},
$$

where $x_{\mathrm{OFF}}$ was the chamber $x$ coordinate offset, and $\mathrm{ADC}_{\mathrm{O}-\mathrm{E}}$ and $\mathrm{ADC}_{0}$ were the $(\mathrm{O}-\mathrm{E})$ ADC signal and associated offset, respectively.

The individual chamber phases $n$ in the Eq. (5) and absolute horizontal coordinate offsets $x_{\mathrm{OFF}}$ and $y_{\mathrm{OFF}}$ between six chambers were adjusted with help of two-dimensional histograms $\left(x_{1}-x_{3}\right)$ vs $\left(x_{2}-x_{3}\right)$ and $\left(y_{1}-y_{3}\right)$ vs $\left(y_{2}-y_{3}\right)[44,45]$.

\section{Raw Data Reduction}

Data summary files were analyzed offline by compressing the DSF event data into PAW Ntuples [46]. Drift time-to-distance lookup tables and assorted chamber calibration constants, as well as CsI detector ADC pedestals and ADC temperature corrections, were determined for each run separately. The high voltages of the CsI detector phototubes were selected to give $\sim 8$ ADC channels/MeV scale. The FWHMs of the pedestal peaks were typically 2 ADC 
channels. Therefore, the pedestal widths corresponded to an energy deposition of $0.25 \mathrm{MeV}, 24 \mu \mathrm{m}$ pathlength or $0.5 \%$ of the most probable energy deposition by a minimum ionizing charged particle. Drifts in the pedestal position over a one month period amounted to less than 2 ADC channels.

The precise horizontal coordinate offsets of six CsI crystals inside the dark box were determined in the next stage of analysis. Using the preliminary offsets read from the plastic template on which the crystals were laying, between 5 and 10 percent of the reconstructed cosmic muon trajectories were found not to intersect any of the predefined detector volumes. After adjusting the $x$ and $y$ translation software offset parameters of the CsI modules by maximizing the number of tracks intersecting individual crystal volumes with nonzero ADCs, the real number of events undergoing scattering in the apparatus or having the improperly reconstructed tracks was shown to be below $1 \%$. That percentage was in agreement with the Monte Carlo simulation of cosmic muons interacting with the experimental apparatus, showing the most probable muon scattering angle of $0.25^{\circ}$ and the $2.5^{\circ}$ mean scattering angle. The Monte Carlo rootmean-square of the scattering angle for the accepted events was $0.66^{\circ}$, Fig. 7 . That value translates into an average pathlength uncertainty of $1 \mathrm{~mm}$. The estimated error in finding the correct crystal position inside the dark box was smaller than $\sim 0.5 \mathrm{~mm}$, Fig. 8 .

The energy deposited in CsI crystal by cosmic muons along the fixed pathlength is a broadened distribution due to the statistical nature of energy transfers. Maximal measured pathlengths were up to $12 \mathrm{~cm}$, while the average pathlength was $\sim 6 \mathrm{~cm}$. A Gaussian model for the energy loss distribution of the minimum ionizing particles in CsI thicknesses of less than $12 \mathrm{~cm}$ is not a good approximation. The distribution of energy losses is asymmetric, with a long high energy tail; the most probable energy loss is smaller than mean energy loss [47].

Light yield temperature coefficients for the individual CsI detectors were determined by the "robust" estimation [48] of the ADC values per unit pathlength as a linear function of temperature recorded inside the dark box. The leastsquares condition assumes normally distributed measurements with constant standard deviations and is therefore not appropriate in this application, as pointed out above. Typically, more than $10^{5}$ cosmic muon events, collected over at least one week and spanning the temperature range of $\sim 2{ }^{\circ} \mathrm{C}$, were used as an input experimental data set. The FORTRAN subroutine MEDFIT, documented in the Ref. [49], was adopted as the fitting procedure by imposing the requirement of minimum absolute deviation between the measured and calculated ADC values per unit path that were dependent linearly on the temperature variable. The average light output temperature coefficient for 74 different CsI detectors extracted by that method was $-1.4 \pm 1.4 \% /{ }^{\circ} \mathrm{C}$, both for fast and total scintillation light components. These numbers are in good agree- 
ment with the previously reported value of $-1.5 \% /{ }^{\circ} \mathrm{C}[11]$, but the spread of extracted coefficients for different $\mathrm{CsI}$ detectors was large: $\pm 4 \% /{ }^{\circ} \mathrm{C}$ (Fig. 9). We point out that the method did not allow us to separate the temperature dependence of the crystal light outputs from the temperature instabilities of the phototubes and high voltage dividers as well as the temperature instabilities of the ADC modules themselves. The LeCroy catalogue specification [50] lists the typical temperature coefficient of a LCR 2249A ADC unit as zero, and the maximum coefficient up to $\pm 3 \%$ for an ADC gate longer than 100 ns and an average ADC reading of $75 \mathrm{pC}$ (about a quarter of a full $256 \mathrm{pC}$ scale).

The raw ADC values were corrected for the temporal temperature variations and written into the revised DSF files used in subsequent analysis.

\section{Detector Photoelectron Statistics}

The contribution of the photoelectron statistics to the energy resolution of CsI crystals was determined by a photodiode-based system. Six individual detectors (CsI crystals with photomultiplier tubes and high voltage dividers) having identical light emitting diodes (LEDs) coupled to their back sides were placed inside the cosmic muon tomography apparatus. The LEDs were pulsed at a $10 \mathrm{~Hz}$ rate using a multichannel diode driver with continuously adjustable output voltage. One split output signal from each channel of the driver generated a 100 ns wide ADC gate. The LED light in a CsI detector produced fast ( $\sim 20$ ns FWHM) PMT anode pulses whose integrated values depended on the driving voltage and were equivalent to the fixed cosmic muon energy depositions between $10 \mathrm{MeV}$ and $100 \mathrm{MeV}$.

A total of five different LED amplitudes were used in measurements of each CsI detector. Examples of the LED spectra are shown in Fig. 10. The ADC pedestals were recorded simultaneously during the run. The intensity of LED light was cross-calibrated against the cosmic muon spectra peaks in CsI crystals. These muons were tracked in tomography drift chambers and both their pathlengths and energy depositions in the crystals could be easily calculated. That calculation enabled the establishment of the absolute energy scale in $\mathrm{MeV}$.

The variance $\sigma_{E}^{2}$ of a photodiode peak depended upon the mean number of photoelectrons $\bar{N}_{p e}$ on the photocathode created per unit energy deposition in the crystal:

$$
\sigma_{E}^{2}=\sum_{i} \sigma_{i}^{2}+\bar{E} / \bar{N}_{p e}
$$


where $\bar{E}$ was the LED spectrum peak position and $\sigma_{i}^{2}$ 's were assorted variances, such as instabilities of the LED driving voltage and temporal pedestal variations. Five measured points $\left(\sigma_{E}^{2}, \bar{E}\right)$ were fitted with a linear function (6) and the mean number of photoelectrons per $\mathrm{MeV} \bar{N}_{\text {pe }}$ for each CsI detector was determined. The statistical error of the least-squares fits was typically less than one photoelecton/MeV. Using the previously extracted light output temperature coefficients all $\bar{N}_{\text {pe }}$ values were scaled to the $18^{\circ} \mathrm{C}$ point. That value was the designed operating temperature of the PIBETA calorimeter.

The number of extracted photoelectrons per $\mathrm{MeV}$ for the fast scintillation component fell into the 20-130 range as shown in Fig. 11. The hexagonal and pentagonal crystals equipped with three inch phototubes averaged 73 photoelectrons/MeV, while the half-hexagonal and veto detectors with two inch phototubes had a mean of 33 photoelectrons/MeV. The $73 / 33$ ratio is very well explained by the $(3 / 2)^{2}$ ratio of photosensitive areas for two different photocathode sizes. The measured photoelectron statistics were in agreement with the $100 \mathrm{~ns}$ ADC gate values of $\bar{N}_{\mathrm{pe}}=(20-260)$ for large $(\sim 10 \mathrm{~cm})$ pure CsI crystals equipped with two or three inch PMTs reported in the past in Refs. [15-17,24].

The quantum efficiency of our bialkali photocathodes for pure CsI scintillation light was $23 \%$ [32], the average light collection probability for our detector shapes $23 \%$ (Sec. 8.2) and the fraction of deposited energy converted into the scintillation light was about $12 \%$. Therefore, starting from the mean number of 73 photoelectrons/MeV, we calculated that $1 \mathrm{MeV}$ energy deposited in the CsI crystal produced on average about $10^{4}$ scintillation photons.

\section{Monte Carlo Description}

\subsection{Tomography System}

Geometrical layout of the experimental apparatus was defined using the GEANT detector description and simulation tools [51]. The active elements of the simulated apparatus were an aluminum box housing six CsI modules, six multiwire drift chambers, two scintillator planes and a layer of lead brick shielding, all shown in Fig. 4. Generated events were muons with the energy, angular and charge distribution of a hard cosmic ray component at sea level. The zenithal angle $\theta_{z}$ distribution of muons at the ground was assumed to be proportional to $\cos ^{2} \theta_{z}$; the momentum spectrum between 0.1 and $10^{3} \mathrm{GeV} / \mathrm{c}$ and the energydependent ratio of number of positive to number of negative cosmic muons was parameterized from the data given in Refs. [29,52]. 
The simulation trigger was defined by requiring a minimum energy deposition of more than $0.2 \mathrm{MeV}$ in each scintillator plane. This threshold was about one-tenth of the minimum-ionizing peak in the triggered scintillator and corresponded to the discriminator level used in the data acquisition electronics.

Penetrating cosmic muons and generated secondary particles were tracked through the apparatus, and energy depositions, pathlengths in CsI crystals and hits in the drift chambers were digitized. All physical processes were turned on in the GEANT programs with the cutoff energies of $0.2 \mathrm{MeV}$ for charged particles and photons. Inspection of simulated energy deposition spectra showed deviations from theoretical Vavilov distributions [47] expected in the planar detector geometries. The differences were caused by multiple scattering in the irregularly shaped crystals, and were particularly prominent for the events with shorter pathlengths close to the crystal edges. The simulated ADC histograms revealed that triggering cosmic muons deposit on average $10.33 \mathrm{MeV} / \mathrm{cm}$ in a CsI detector, with the most probable energy loss $5.92 \mathrm{MeV} / \mathrm{cm}$.

Cosmic muon spectra in CsI detectors were described in a satisfactory way $\left(\chi^{2} /(m-1) \approx 1.2\right)$ by the combination of a Gaussian distribution and a falling exponential tail:

$$
A(\epsilon)=\theta\left(\epsilon_{0}-\epsilon\right) e^{-\frac{1}{2}\left[(\epsilon-\bar{\epsilon}) / \sigma_{\epsilon}\right]^{2}}+\theta\left(\epsilon-\epsilon_{0}\right) e^{\alpha-\beta \epsilon}
$$

where $\epsilon$ is energy (in MeV) deposited in one full CsI module. Parameters $\bar{\epsilon}, \sigma_{\epsilon}$, $\epsilon_{0}, \alpha$, and $\beta$ are extracted from the GEANT spectra by imposing the least squares constraint to the fit and leaving the pathlength $d(\mathrm{~cm})$ as free parameter:

$$
\begin{aligned}
\bar{\epsilon}(d) & =5.079+0.1876 d-9.9390 \cdot 10^{-3} d^{2} \\
\sigma_{\epsilon}(d) & =0.3545+7.2329 \cdot 10^{-3} d-3.4148 \cdot 10^{-4} d^{2} \\
\epsilon_{0}(d) & =5.1800+0.1989 d-1.0297 \cdot 10^{-2} d^{2} \\
\alpha(d) & =4.0581+0.4465 d-1.5760 \cdot 10^{-2} d^{2} \\
\beta(d) & =0.7952+5.0313 \cdot 10^{-1} d-1.5240 \cdot 10^{-3} d^{2}
\end{aligned}
$$

and

$$
\theta\left(\epsilon_{0}-\epsilon\right)= \begin{cases}0, & \text { if } \epsilon_{0}<0 \\ 1, & \text { otherwise }\end{cases}
$$

These Monte Carlo spectra, broadened with photoelectron statistics, were used to describe our cosmic muon lineshapes produced by the optically uniform CsI detectors. 


\subsection{Light Collection Simulation: TkOptics Code}

Propagation of scintillating photons through a uniform detector with ideal reflecting dielectric surfaces and different wrapping materials was studied using the TkOptics simulation program [53,54]. The code is a library of FORTRAN subroutines with an X-Windows-based user interface written with Tck/Tk toolkit [55]. The program can simulate the light output response of an arbitrarily shaped scintillation detector with given bulk and surface optical properties.

The polygonal detector shape is defined by the coordinates of its vertices. The detector attributes are reflector types of lateral and front crystal surfaces and wrapper material, crystal surface-wrapper gap distances and characteristics of the photomultiplier tube and a phototube-crystal joint coupling. The program handles normal dielectric, specular, diffuse and partially absorbent reflector surfaces with arbitrary diffuse fraction, roughness, and specular $r_{s}$ and diffuse reflectivity $r_{d}$. Predefined bulk properties of a detector are the medium scattering and attenuation length as well as refractive index. A photomultiplier tube is specified by the diameter, position and quantum efficiency of the photocathode.

Different choices of initial scintillating photon distribution are possible, the default being a uniform starting distribution throughout the scintillating volume. A working volume is a box divided into elementary cubic cells of fixed size. Output menu options include the initial and endpoint photon coordinates, and direction vectors and timing distributions of the detected scintillation photons organized in a PAW Ntuple. Every elementary cell is flagged as a bulk or edge crystal cell. Center coordinates of the cells and a fraction of photons generated in every cell, and subsequently detected on the PMT sensitive surface, are always recorded. Results of high statistics runs with $10^{7}$ photons generated uniformly through the detector volume were plotted to show the number of photoelectrons as a function of scintillation source position inside the crystal. The relative statistical errors of calculated light collection probabilities for the bulk crystal cells were $\leq 2 \%$.

The bulk attenuation length and the light scattering length of the nearultraviolet emission component inside the CsI crystals were set to $150 \mathrm{~cm}$ and $200 \mathrm{~cm}$, respectively [56]. The index of refraction for the CsI medium increases from 1.82 for the blue-green light to about 2.08 for ultraviolet light [56].

The simulated light collection probabilities $P(x, z)$ that depend on axial $z$ and transverse $x$ positions are shown integrated in the vertical coordinate $(y)$ in Figs. 12 and 13. in the form of "lego" plots for one wrapping configuration and two different crystal shapes. The coordinate system is defined with the $z=0 \mathrm{~cm}$ origin at the front face of the crystal and a photocathode window at 
the $z=22 \mathrm{~cm}$ plane.

We find that simulated light response of the ideal hexagonal or pentagonal PIBETA CsI detector with specular lateral surfaces and a diffuse wrapping material can be described with the following parameters:

(1) The average photon collection probability $P$ for a three inch photocathode is about $23 \%$ for ideal $r_{s}=1.0$ crystal surfaces and a $r_{d}=0.9$ diffuse wrapper, decreasing by half, to $12 \%$ for $r_{s}=0.8$.

(2) The axial light collection probability variation through the first 10 centimeters is in the $\pm 5 \%$ range, with a positive slope $d P / d z \geq 0$ for higher specular and diffuse reflectivities, namely for $r_{s, d} \geq 0.9$.

(3) The axial detected light variation in the back crystal half $(z \geq 10 \mathrm{~cm})$ is up to $-30 \%$.

(4) The transverse light response referenced to the light yield at the crystal axis typically increases towards the crystal surfaces by up to $+5 \%$ for $z \leq 10 \mathrm{~cm}$, but is generally declining away from the central axis by $-30 \%$ at the $z=18 \mathrm{~cm}$ plane.

(5) The root-mean-square of a three-dimensional light nonuniformity is between $2.5 \%$ and $3.5 \%$ for $z \leq 10 \mathrm{~cm}$, compared to a $\sim 20 \%$ root-meansquare for a $z \geq 10 \mathrm{~cm}$ volume, where a large spread is caused by the inefficient light collection from crystal back corners.

For lateral surfaces painted with a $r_{d} \approx 0.9$ diffuse substance without an air gap we find that the average photon collection probability is lower, $\sim 17 \%$, and the axial light collection nonuniformity as a function of axial position is always positive and is usually larger than $+10 \%$ in a $22 \mathrm{~cm}$ long detector, making the root-mean-square of the $3 \mathrm{D}$ nonuniformity function $\geq 20 \%$.

The Monte Carlo results for the optically uniform half-hexagonal and trapezial CsI crystals with the same range of optical properties predict smaller light yields and somewhat higher light collection nonuniformities:

(1) The average photon collection probability with a two inch photocathode is about $12 \%$.

(2) The typical axial nonuniformity is positive in front, $+5 \% / 10 \mathrm{~cm}$, and negative in the back crystal half, with a $-30 \% / 10 \mathrm{~cm}$ variation,

(3) The simulated transverse light output from the front crystal half is very similar to one for the full crystal shapes, increasing up to $+3 \%$ away from the detector central axis, but decreasing by almost a third in the back corners of the crystal.

(4) The root-mean-square of detected light output varying between $3.0 \%$ and $4.5 \%$, in the front $(z \leq 10 \mathrm{~cm})$ and back $(z \geq 10 \mathrm{~cm})$ crystal halves, respectively.

These light collection probability distributions calculated for optically homo- 
geneous crystals explain the major features of the measured optical nonuniformities presented below.

\section{Measured Nonuniformities of CsI Detector Light Responses}

The three-dimensional (3D) spatial distribution of the light output of a scintillation detector can be specified by giving the number of photoelectrons $N_{\text {pe }}(x, y, z)$ produced by $1 \mathrm{MeV}$ energy deposition at point $(x, y, z)$ ("3D light nonuniformity function"). In the following discussion we limit ourselves to the linear one-dimensional variations of the detected light output separable in the axial and transverse directions:

$$
N_{\mathrm{pe}}(z, x) \propto\left\{\begin{array}{l}
N_{1}+a_{z 1} \cdot z+a_{t}(z) \cdot x, \quad z \leq 10 \mathrm{~cm}, \text { and } x= \pm 15 \mathrm{~cm} \\
N_{2}+a_{z 2} \cdot z+a_{t}(z) \cdot x, \quad z \geq 10 \mathrm{~cm}, \text { and } x= \pm 15 \mathrm{~cm}
\end{array}\right.
$$

where $a_{z}\left(a_{t}\right)$ is the linear optical nonuniformity coefficient in the $z(x)$ coordinate, and the coordinate system origin is at the center of the detector front face, as explained in Sec. 8.2.

A simple and straightforward parameterization of the detector light response nonuniformity can be made on the basis of scatter plots showing the light output per unit pathlength as a function of longitudinal or transverse cosmic muon-CsI crystal intersection coordinates. In this analysis we take only cosmic muon events with almost perpendicular trajectories $\left(\theta_{z} \geq 85^{\circ}\right)$, so that the measured two-dimensional light outputs are averaged over the pathlengths and over $\sim 1 \mathrm{~cm}^{2}$ vertical cross sections. The cosmic muons deposit the energy along the well-defined ionization track of the length $d$ in the crystal. This description will somewhat underestimate the real light collection probabilities (see Sec. 10) due to the integration of a three-dimensional light nonuniformity function along the charged particle track. Our work on the fully 3D reconstruction of the scintillator light response will be presented in a forthcoming publication [57].

Fig. 14 shows the axial variation of normalized ADC values as a function of distance of energy deposition from the front crystal face for the six representative crystals. The axial positions were calculated by averaging two $z$ values of the cosmic muon track intersection with the crystal surfaces.

Panels on Figs. 15 and 16 show the transverse dependence of measured light output per unit pathlength, where the independent variable is the distance from the crystal axis measured in the horizontal plane. The selected scatter plots show the data points for the axial slices at $z_{0}=6 \pm 1 \mathrm{~cm}$ and $z_{0}=18 \pm 1 \mathrm{~cm}$. 
We have chosen to parameterize the axial light output nonuniformity with two piecewise linear functions: the light output nonuniformity coefficient $a_{z 1}(z \leq 10 \mathrm{~cm})$ for the front half of the crystal, and the light nonuniformity coefficient $a_{z 2}(z \geq 10 \mathrm{~cm})$ for the back half of the crystal, both values expressed in $\% / \mathrm{cm}$. The transverse light output variation at a fixed axial distance $z_{0}, a_{t}\left(z_{0}\right)$, is described by the average value of a change in luminosity left and right of the crystal axis.

Several general features are readily noticeable: the spread of measured points due to energy deposition straggling, the gradual variation in the collected light along and perpendicular to the detector axis, and the decrease in detected light when the photon generation occurs close to lateral detector surfaces and, in particular, near the back corners of the detector volumes.

The calculated ADC/pathlength data points shown on the panels of Figs. 1416 have been fitted with linear functions imposing the "robust" condition of the minimum absolute deviation between the measured and calculated values, Eqs. 14. The normalized light output scatter plots in the axial and transverse coordinates, as well as two-dimensional $\mathrm{ADC} / d$ distributions in the $x-z$ bins and associated linear light output nonuniformity coefficients have been documented for all studied CsI crystals and are available for inspection at the PIBETA WWW site [58]. The average scintillation properties of all the measured CsI crystals are listed in Tables 1 and 2.

In summary, for a set of fifty-nine full hexagonal and pentagonal crystals we find:

(1) The average axial light nonuniformities are $a_{z 1}(z \leq 10 \mathrm{~cm})=-0.1 \% / \mathrm{cm}$ and $a_{z 2}(z \geq 10 \mathrm{~cm})=-1.3 \% / \mathrm{cm}$, respectively (see Fig. 17).

(2) Distribution of nonuniformity coefficients could be described by a Gaussian with $\sigma_{a_{z 1}} \approx 1.3 \% / \mathrm{cm}$.

(3) The average transverse light output is flat for $z \leq 10 \mathrm{~cm}$, and the typical light variation is $-15 \%$ at $z=18 \mathrm{~cm}$.

(4) The Kharkov-grown crystals on average have twice the axial optical nonuniformity of the Bicron-grown crystals.

For fifteen half-hexagonal and trapezial crystal shapes we find:

(1) The average axial nonuniformities are $a_{z 1}(z \leq 10 \mathrm{~cm})=-0.3 \% / \mathrm{cm}$ and $a_{z 2}(z \geq 10 \mathrm{~cm})=-1.0 \% / \mathrm{cm}$.

(2) The transverse light output variation is limited in the front crystal half, but increases up to $-30 \%$ in the back of the crystal.

With the insights gained in the simulation of the light collection probabilities (Sec. 8.2) we conclude that the $\pm 2 \% / \mathrm{cm}$ spread of the nonuniformity coefficients corresponds to an equivalent range of 0.8-1.0 in crystal surface reflectivities, and/or 100-250 cm range in CsI attenuation lengths. 


\section{Light output uniformity scans of CsI crystals with ${ }^{137}$ Cs gamma source}

A light-tight plywood box was made to house a single CsI detector and associated measurement apparatus described below. A $0.662 \mathrm{MeV}{ }^{137} \mathrm{Cs}$ gamma source was placed on a moveable support next to the detector and collimated by a $20 \times 10 \times 5 \mathrm{~cm}^{3}$ lead brick with a $6 \mathrm{~mm}$ collimator hole. The photomultiplier analog signal from the detector was processed with an ORTEC 454 timing amplifier with a gain of 30 and a 50 ns integration time constant. The amplifier output was digitized with a peak sensing ORTEC AD811 ADC unit. The same signal was discriminated and produced the trigger rate of about $5 \mathrm{kHz}$ with a ${ }^{137} \mathrm{Cs}$ source present. The background rate, without the source present, was 50-100 Hz.

Pedestal runs were taken with a clock trigger and used to properly correct offsets of the ADC spectra. Temperature variation during one run was less than $0.3^{\circ} \mathrm{C}$, typically causing the overall light output variation of less than $0.5 \%$ in a ADC spectrum that was gated with a 100 ns window. Several runs were taken with the source removed to find the shape of the background spectrum. It was confirmed that the background spectrum does not depend on the position of the lead collimator, so the same background lineshape was used in the analysis for all source positions.

The collimated ${ }^{137} \mathrm{Cs}$ source was placed by remote control in turn at five points along the axis of each crystal at 2, 6, 10, 15 and $20 \mathrm{~cm}$ from the front face of the crystal.

Following background subtraction, recorded spectra were fitted with a sum of a Gaussian and exponential function, Fig. 18. The peak position and the FWHM for each spectrum were extracted with the statistical uncertainty lower than $0.3 \%$.

The dependence of the peak position on the placement of the source is illustrated in Fig. 19 and follows the trend of the tomography results. The GEANT simulations revealed that the $662 \mathrm{keV}$ gamma rays could probe all the volume of CsI crystal, but the shower energy deposited at the central axis is only about one-sixth of the energy deposition near the crystal surface. The cosmic muons transfer the energy uniformly along their tracks in CsI material: the scintillation volume over which the measured light output is integrated is therefore larger, and inherent averaging leads to smaller extracted optical nonuniformity coefficients. This feature is borne out in the panels of Fig. 18 where the tomography data for three different crystals yield consistently smaller axial light nonuniformities when compared with the radioactive source measurements.

The simple ${ }^{137} \mathrm{Cs}$ scans with the described apparatus were used to evaluate 
the light collection nonuniformities for up to six crystals per day.

Radioactive source scan measurements similar to ours are described in Refs. [22,31].

\section{A GEANT simulation of the PIBETA calorimeter response to 10- $120 \mathrm{MeV}$ positrons and photons}

We have studied the simulated PIBETA calorimeter response to the $69.8 \mathrm{MeV}$ $\pi^{+} \rightarrow e^{+} \nu$ positrons and to the $\left(69.8+2 m_{e^{+}}\right) \mathrm{MeV}$ photons, where $m_{e^{+}}$is the positron rest mass. The goal was to find the intrinsic difference between the responses to monoenergetic positrons and photons with identical total incident energies, emphasizing the correct modeling of the low-energy tail below the edge of the Michel $\mu \rightarrow e^{+} \nu \nu$ spectrum at $52.8 \mathrm{MeV}$.

The $0.46 \%$ accuracy of the new TRIUMF measurement [4] of the $\pi^{+} \rightarrow e^{+} \nu$ branching ratio was limited by event statistics and systematic uncertainties in evaluating the low-energy tail of the positron peak. The $\pi^{+} \rightarrow e^{+} \nu \gamma$ positrons and the forward-peaked bremsstrahlung gammas were detected in a 46-cm-diameter $\times 51$-cm-long $\mathrm{NaI}(\mathrm{Tl})$ crystal "TINA". The tail correction of $1.44 \pm 0.24 \%$ for the energy region from $0 \mathrm{MeV}$ to $52.3 \mathrm{MeV}$ was determined by subtracting the measured Michel positron spectrum from the detector positron response functions measured with the $20-85 \mathrm{MeV} e^{+}$monoenergetic beams. An additional tail component of $\sim 0.4 \%$ due to radiative processes was estimated by Monte Carlo method. The TRIUMF group apparently made no attempt to account for the potential difference in the scintillator light response of positrons and photons and neglected potential nonlinearities of the energy scale. Both effects could arise from the light collection nonuniformities of the $\mathrm{NaI}(\mathrm{Tl})$ detector.

In the recent PSI $\pi^{+} \rightarrow e^{+} \nu$ experiment [3] the quoted $0.29 \%$ systematic uncertainty of the extracted branching ratio was also dominated by the $1.64 \pm 0.09 \%$ electromagnetic loss fraction as well as by the $0.95 \pm 0.19 \%$ contribution from the photonuclear reactions. The $\sim 4 \pi$ calorimeter used in that measurement was made of 132 hexagonally shaped BGO crystals, with the light yield claimed to be homogeneous to within $1.5 \%$ over the whole $20 \mathrm{~cm}$ length of every crystal.

The lineshapes of positrons and photons in our PIBETA calorimeter and their dependence on the optical properties of 240 constituent CsI crystals were predicted using the GEANT detector description and simulation tools. The code defined the geometry and tracking media for the complete PIBETA detector [59]. The inner detector region was occupied by two cylindrical wire chambers and a segmented plastic veto detector. The incident particles, monoener- 
getic positrons and photons, were generated in the center of the crystal sphere.

Photonuclear reactions in the active detectors as well as in the surrounding passive material were modeled in a user subroutine [60] that was added to the default GEANT version 3.21. The probabilities of photonuclear reactions were calculated using the published cross sections from the $\gamma N$ reaction thresholds up to the energy of $120 \mathrm{MeV}$ [61-66].

The 240 individual CsI module shapes were specified, taking into account the mechanical tolerances of the physical CsI crystals, with Teflon and aluminized Mylar wrappings filling the $200 \mu \mathrm{m}$ intermodule gaps. The irregular CsI modules had to be constructed from up to six GEANT generalized trapezoidal wedges. Every CsI crystal volume was considered a sensitive detector with the associated luminosity $\bar{N}_{\text {pe }}$ expressed as the number of photoelectrons per $\mathrm{MeV}$, and two axial and one transverse light collection nonuniformity coefficients, $a_{z 1}, a_{z 2}$, and $a_{t}$, respectively. A set of seventy-four detector luminosity and nonuniformity values, extracted in the tomography analysis, were initialized in the GEANT-accessible database. The optical parameters of the remaining 163 modules were drawn randomly from the distributions in Figs. 11 and 17. The assumption was that the crystals produced in the future will be of the same optical quality as the ones that are already delivered.

We have always simulated at least $10^{5}$ events for every fixed set of the calorimeter parameters $\left(\bar{N}_{\mathrm{pe}}, a_{z 1}, a_{z 2}, a_{t}\right)$. The low-threshold trigger was defined by requiring a sum of the $\mathrm{ADC}$ readings exceed $5 \mathrm{MeV}$ of the light-equivalent energy for one calorimeter supercluster containing 32 crystals. The integrated detector acceptances with a low-level trigger were $85.8 \%$ for $\sim 70 \mathrm{MeV}$ positrons and $85.5 \%$ for $\sim 70 \mathrm{MeV}$ photons, so all extracted quantities had the relative statistical uncertainties of $\sim 0.2 \%$. The response of the calorimeter was parameterized by the FWHM of the simulated ADC spectrum and the tail contribution being between the adjustable low level (default LT being $5 \mathrm{MeV}$ ) and high level thresholds (two default values $\mathrm{HT}_{1}$ and $\mathrm{HT}_{2}$ being $54 \mathrm{MeV}$ and $55 \mathrm{MeV}$, respectively).

For the purposes of comparison and gain normalization we first studied the response of an ideal, homogeneous calorimeter, with realistic CsI crystal light outputs. The "software" gain factor of every individual CsI detector was determined from the fitted peak positions of the simulated ADC spectrum sum over the crystal with maximum energy deposition and its nearest neighbors. The peak value positions were found after smoothing the histograms with a multiquadric function, to improve on the limited simulation statistics. The ratios of peak positions in the ideal, homogeneous calorimeter and the corresponding values for the physical, nonuniform calorimeter, were, by definition, the individual detector gain corrections. The values of these ratios were refined in three steps of the iterative procedure. The extracted energy resolution of 
the nonuniform detector in principle depends on the convergence of the gainmatching process and the resulting uncertainties of the individual detector gains. That gain-matching process was equivalent to the PMT high voltage adjustments in the real experiment that are effected to obtain the best energy resolution with the modular detector. We estimate that our simulation procedure fixes the software detector gains with the accuracy of $\sim 1 \%$.

The set of gain constants depended on the optical properties of our CsI crystals as defined in our GEANT database, but also upon the incident particle chosen for the Monte Carlo calibration runs and its energy because of differences in shower developments of photons and positrons. The gain normalizations of the calorimeter detectors were calculated by aligning the simulated $\pi^{+} \rightarrow e^{+} \nu$ positron peak positions. The same procedure for matching the CsI detector gains was used in the real data-taking runs. Due to the gain renormalization, the influence of transverse light nonuniformities smaller than $10 \%$ on the ADC spectrum peak positions and the ADC lineshape could be neglected in comparison with the axial light nonuniformity effects. The simulated ADC values were summed over all detectors with the energy deposition above the $1 \mathrm{MeV}$ TDC threshold. We also examined the calculated ADC sums for the clusters which contained the crystal with the maximum energy deposition and its nearest neighbors. The full-widths at half maximum for these spectra are labeled in the following figures and tables as $\mathrm{FWHM}_{(220)}$ and $\mathrm{FWHM}_{(\mathrm{NN})}$, respectively. No event-to-event uncertainties of the ADC pedestal values were assumed in the simulation. Stability of the PIBETA electronics tested under real experimental conditions in 1996 calibration runs and the quality of the algorithms used for the first and second pedestal correction reduces the pedestal peak root-mean-square to $\sim 8$ channels, equivalent to $\sim 0.3 \mathrm{MeV}$.

The GEANT-calculated resolution of the PIBETA calorimeter consisting of 220 CsI detector crystals and 20 veto crystals was parameterized by the fractional full width at half maximum:

$$
\begin{aligned}
\frac{\operatorname{FWHM}_{(22)}}{E_{e^{+}}}(\%) & =\frac{2.36 \cdot \sigma_{E_{e^{+}}}}{E_{e^{+}}}= \\
& =\left\{\begin{array}{l}
\left(54.69+1.197 E_{e^{+}}+0.4656 \cdot 10^{-2} E_{e^{+}}^{2}\right) / E_{e^{+}}^{0.8030} \\
\left(37.19+0.060 E_{e^{+}}+0.2734 \cdot 10^{-2} E_{e^{+}}^{2}\right) / E_{e^{+}}^{0.5256}
\end{array}\right.
\end{aligned}
$$

for the positrons with the most probable peak energy $E_{e^{+}} \mathrm{MeV}$ and

$$
\frac{\mathrm{FWHM}_{(220)}}{E_{\gamma}}(\%)=\left\{\begin{array}{l}
\left(37.07+0.5507 E_{\gamma}+0.3233 \cdot 10^{-2} E_{\gamma}^{2}\right) / E_{\gamma}^{0.6549} \\
\left(40.52+0.9437 \cdot E_{\gamma}+0.4931 \cdot 10^{-2} E_{\gamma}^{2}\right) / E_{\gamma}^{0.6942}
\end{array}\right.
$$

for the monoenergetic photons with the detected peak energy $E_{\gamma} \mathrm{MeV}$ (Fig. 20). The top lines refer to the cases of an optically uniform detector $\left(a_{z}=0\right)$ while 
the bottom equations describe the predicted response of the nonuniform calorimeter $\left(a_{z}=\right.$ TOMOGRAPHY $)$.

The average fractional energy resolution $\mathrm{FWHM}_{(220)}$ of $5.3 \%(6.0 \%)$ was achieved for the $\sim 70 \mathrm{MeV}$ kinetic energy positrons in the optically uniform (nonuniform) calorimeter, as compared to $5.7 \%$ (6.8\%) resolution for the equivalent energy photons. That was the resolution found with no cuts applied on the light-equivalent energy generated in the calorimeter vetoes. Requiring less than $5 \mathrm{MeV}$ detected in the veto shield decreased the statistics by about $10 \%$ and suppressed the low-energy tail, but did not improve the energy resolution at the peak position.

Limiting the ADC sums to the nearest-neighbor crystals (six or seven crystal clusters) the fractional $\mathrm{FWHM}_{(\mathrm{NN})}$ energy resolution could be parameterized by:

$$
\frac{\mathrm{FWHM}_{(\mathrm{NN})}}{E_{e^{+}}}(\%)=\left\{\begin{array}{l}
\left(69.19+3.057 E_{e^{+}}+0.4198 \cdot 10^{-2} E_{e^{+}}^{2}\right) / E_{e^{+}}^{0.8560} \\
\left(68.13+2.479 E_{e^{+}}+0.3368 \cdot 10^{-2} E_{e^{+}}^{2}\right) / E_{e^{+}}^{0.8018}
\end{array}\right.
$$

for the positrons with the detected peak energy $E_{e^{+}} \mathrm{MeV}$ and

$$
\frac{\mathrm{FWHM}_{(\mathrm{NN})}}{E_{\gamma}}(\%)=\left\{\begin{array}{l}
\left(43.16+2.318 E_{\gamma}+0.4148 \cdot 10^{-2} E_{\gamma}^{2}\right) / E_{\gamma}^{0.7723} \\
\left(39.57+2.302 E_{\gamma}+0.1329 \cdot 10^{-2} E_{\gamma}^{2}\right) / E_{\gamma}^{0.7199}
\end{array}\right.
$$

for the monoenergetic photons with the detected peak energy $E_{\gamma} \mathrm{MeV}$ (Fig. 21).

The average $\mathrm{FWHM}_{(\mathrm{NN})}$ for the $70 \mathrm{MeV}$ positrons and gammas in nearestneighbor nonuniform crystal clusters was $8.7 \%(6.1 \mathrm{MeV})$ and $9.9 \%(6.8 \mathrm{MeV})$, respectively. Imposing the $5 \mathrm{MeV}$ cut on the detector veto signals improves these resolutions only marginally. These numbers should be compared with the response width of the ideal uniform nearest-neighbor clusters: $8.2 \%(5.7 \mathrm{MeV})$ and $8.7 \%(6.2 \mathrm{MeV})$.

The percentage of the events in the tail between the preset low and high energy threshold was tracked in the same GEANT simulation.

The listing of the low-energy tail contributions for $69.8 \mathrm{MeV} e^{+}$and $70.8 \mathrm{MeV} \gamma$ in Table 3 shows that in the optically uniform calorimeter they differ by $1.8 \%$. The optical nonuniformity does not change the positron tail contribution, but increases the photon tail by $\sim 0.2 \%$. The imposition of the $5 \mathrm{MeV}$ veto-shield cut decreases the electromagnetic leakage to $2.4 \%$ for positrons and $4.7 \%$ for photons, a $e^{+}-\gamma$ tail difference of $+2.3 \%$.

Table 4 shows the low-energy tail components for the simulated nearestneighbor cluster ADC sums: with no applied cuts the contributions were $4.7 \%$ 
and $6.7 \%$ for $e^{+}$'s and $\gamma^{\prime}$ s, respectively. The veto shielding cuts decreased both fractions by about $0.3 \%$.

If the positron originates from a radiative $\pi^{+} \rightarrow e^{+} \nu \gamma$ decay, its average Monte Carlo peak position is essentially unchanged. Its tail contribution is unaffected if simulated ADC sums extend over full calorimeter but are increased by $1.4 \%$ if only the nearest-neighbor clusters are summed. The radiative decay matrix elements used in the calculation were taken from Ref. [67]. All quoted numbers have an approximate systematic uncertainty of $\sim 0.2 \%$.

The detector response to positrons and photons in the energy range between $10 \mathrm{MeV}$ and $120 \mathrm{MeV}$ is also nonlinear because the volume distribution of the shower energy deposition depends on the incident particle type and the energy. Our GEANT studies showed that the magnitude and energy dependence of these nonlinearities does not change significantly because of CsI crystal light collection nonuniformity. Results of the calculations, both for the case of a homogeneous and optically nonhomogeneous calorimeter, are displayed in Figs. 22 and 23. Nonlinearities of the detector response in the covered energy range for positrons and photons are very close in magnitude and shape of energy variation and amounted to $\sim 1.5 \%$ for the ADC sums over 220 crystals and up to $\sim 2.8 \%$ if the simulated ADC sums were restricted to the over-the-threshold ADCs of nearest neighbors.

\section{Conclusion}

We have measured the optical properties of seventy-four pure cesium iodide crystals that were polished and wrapped in the diffuse Teflon reflector. The results are summarized separately for the full-sized and half-sized crystals in Tables 1 and 2 .

The deduced light yields parameterized by two axial and one transverse light collection nonuniformity coefficients constitute a minimum set of parameters necessary for a realistic Monte Carlo simulation of the modular CsI calorimeter.

The predicted energy resolutions $\mathrm{FWHM}_{(220)}$ for $\sim 70 \mathrm{MeV}$ positrons and photons in the full PIBETA calorimeter with the ideal, optically uniform CsI modules with specified luminosities were shown to be close, $3.7 \mathrm{MeV}$ and $4.0 \mathrm{MeV}$, respectively. The upper limit of the low energy tail contributions in the region between $5 \mathrm{MeV}$ and $55 \mathrm{MeV}$ were calculated to be $6.9 \%$ and $8.7 \%$ for the positrons and gammas in an optically homogeneous detector, respectively. After applying the $5 \mathrm{MeV}$ calorimeter veto cut, these tail corrections decrease to $2.2 \%$ and $4.5 \%$. 
The average deduced axial light nonuniformities of real CsI crystals wrapped in a Teflon sheet had negative slopes, $-0.18 \% / \mathrm{cm}$ and $-1.6 \% / \mathrm{cm}$, for the front and back half of the crystal volume, respectively. The corresponding GEANT simulation of the nonuniform PIBETA apparatus for $68.9 \mathrm{MeV} e^{+}$and $70.8 \mathrm{MeV} \gamma$ 's shows that both energy responses will be broadened to the average $\operatorname{FWHM}_{(220)}$ of $5.9 \%$ and $6.9 \%$ while the associated tail contributions will change only for photon spectra, increasing the low-energy tail by $\sim 0.3 \%$. The simulated calorimeter ADC spectra are shown in Figs. 24 and 25.

The nonlinearity of the measured energy scale caused by the optical nonuniformity is $\leq 2.8 \%$ throughout the relevant $e^{+} / \gamma$ energy range. This spread is consistent with the precision of energy calibration required to extract the tail corrections with the systematic uncertainty of $\sim 0.2 \%$.

The predicted ADC spectra of the monoenergetic positrons, electrons and tagged photons in the energy range $10-70 \mathrm{MeV}$ will be compared with the measured responses of the partial CsI calorimeter arrays in a forthcoming publication [68].

\section{Acknowledgements}

The authors wish to thank Micheal Sadler of the Abilene Christian University (ACU) for lending us the drift chamber tomography apparatus. Derek Wise, also of ACU, has helped with the cosmic muon tomography measurements. Roger Schnyder of Paul Scherrer Institut has maintained and repaired the faulty electronics modules. Their help is gratefully acknowledged.

This work is supported and made possible by grants from the US National Science Foundation and the Paul Scherrer Institute. 


\section{References}

[1] D. Počanić, D. Day, E. Frlež, R. M. Marshall, J. S. McCarthy, R. C. Minehart, K. O. H. Ziock, M. Daum, R. Frosch and D. Renker, PSI R-89.01 Experiment Proposal (Paul Scherrer Institute, Villigen, 1988).

[2] W. K. McFarlane, L. B. Auerbach, F. C. Gaille, V. L. Highland, E. Jastrzembski, R. J. Macek, F. H. Cverna, C. M. Hoffman, G. E. Hogan, R. E. Morgado and R. D. Werbeck, Phys. Rev. D32 (1985) 547.

[3] G. Czapek, A. Federspiel, A. Fluckiger, D. Frei, B. Hahn, C. Hug, E. Hugentobler, W. Krebs, U. Moser, D. Muster, E. Ramseyer, H. Scheidiger, P. Schlatter, G. Stucki, R. Abela, D. Renker and E. Steiner, Phys. Rev. Lett. 70 (1993) 17.

[4] D. I. Britton, S. Ahmad, D. A. Bryman, R. A. Burnham, E. T. H. Clifford, P. Kitching, Y. Kuno, J. A. Macdonald, T. Numao, A. Olin, J. M. Poutissou and M. S. Dixit, Phys. Rev. D49 (1994) 28.

[5] A. Sirlin, Rev. Mod. Phys. 50 (1978) 573.

[6] A. Sirlin, private communication (1989).

[7] I. S. Towner and J. C. Hardy, Currents and Their Couplings in the Weak Sector of the Standard Model, NUCL-TH/9504015 Preprint (1995).

[8] W. J. Marciano and A. Sirlin, Phys. Rev. D35 (1987) 1672.

[9] R. B. Murray and F. J. Keller, Phys. Rev. 137A (1965) A942.

[10] C. W. Bates, A. Salau, and D. Leniart, Phys. Rev. B15 (1977) 5963.

[11] H. Kobayashi, A. Konaka, K. Miyake, T. T. Nakamura, T. Nomura, N. Sasao, T. Yamashita, S. Sakuragi, and S. Hashimoto, Kyoto University Preprint KUNS900 (1987).

[12] S. Kubota, H. Murakami, J. Z. Ruan, N. Iwasa, S. Sakuragi, and S. Hashimoto, Nucl. Inst. and. Meth. A273 (1988) 645.

[13] S. Kubota, S. Sakuragi, S. Hashimoto and J. Z. Ruan, Nucl. Inst. and Meth. A268 (1988) 275.

[14] S. Keszthelyi-Lándori, I. Földvári, R. Voszka, Z. Fodor, and Z. Seres, Nucl. Inst. and Meth. A303 (1991) 374.

[15] C. L. Woody, P. W. Levy, J. A. Kierstead, T. Skwarnicki, Z. Sobolewski, M. Goldberg, N. Horwitz, P. Souder and D. F. Anderson, IEEE Trans. Nucl. Sci. 37 (1990) 492.

[16] P. Schotanus, R. Kamermans and P. Dorenbos, IEEE Trans. Nucl. Sci. 37 (1990) 177.

[17] B. K. Utts and S. E. Spagno, IEEE Trans. Nucl. Sci. 37 (1990) 134. 
[18] A. V. Gektin, A. I. Gorelov, V. I. Rykalin, V. I. Selivanov, N. V. Shiran, and V. G. Vasilchenko, Nucl. Inst. and Meth. A294 (1990) 591.

[19] M. M. Hamada, Y. Nunoya, S. Kubota, and S. Sakuragi, Nucl. Inst. and. Meth. A365 (1995) 98.

[20] C. Woody, BNL-47776-MC (1992).

[21] Z. Y. Wei and R. Y. Zhu, Nucl. Inst. and Meth. A326 (1993) 326.

[22] J. Brose, G. Dahlinger, P. Eckstein, K. R. Schubert and R. Seitz, SLAC BaBar Note \#175, Stanford (1995).

[23] G. Dahlinger, SLAC BaBar Note \#241, Stanford (1996).

[24] C. Morris and R. L. Boudrie, A Proposal for a High Resolution Spectrometer for Neutral Mesons (LAMPF, Los Alamos, 1989).

[25] R. E. Ray, FERMILAB-CONF-94-418 (1994).

[26] R. S. Kessler, A. Roodman, P. Shawhan, N. Solomey, B. Winstein, S. Hansen, H. Nguyen, R. Ray, R. Tschirhart, J. Whitmore, T. Nakaya, and M. Lindgren, Nucl. Inst. and Meth. A368 (1996) 653.

[27] G. David, A. Hershcovitch, S. Stoll, C. Woody, P. B. Munzinger, R. Hutter, J. Stachel, C. M. Zou, N. Horwitz, and Z. Sobolewski, Nucl Inst. and Meth. A348 (1994) 87.

[28] H. Kenner, Geodesic Math and How to Use It (University of California Press, Berkeley, 1976).

[29] Review of Particle Physics, Particle Data Group, Phys. Rev. D54 (1996) 72.

[30] K. A. Assamagan, Ph. D. Thesis (University of Virginia, Charlottesville, 1995) unpublished.

[31] D. H. Dowell, A. M. Sandorfi, A. Q. R. Baron, B. J. Fineman, O. C. Kistner, G. Matone, C. E. Thorn, and R. M. Sealock, Nucl. Instr. and Meth. A286 (1990) 183.

[32] THORN-EMI Electron Tubes Catalog: Photomultipliers and Accessories, Rockaway, NJ (1993).

[33] C. L. Smith, PMT Evaluation PIBETA Note, accessible at URL http://helena.phys.virginia.edu/ pibeta/ or http://psw340.psi.ch/ pibeta/, (1995).

[34] E. Frlež, Ch. Brönnimann, T. Flügel, J. E. Koglin, B. Krause, D. Počanić, D. Renker, S. Ritt, P. L. Slocum, The Light Response of Pure CsI Calorimeter Crystals Painted with Waveshift Lacquer, to be submitted to Nucl. Phys. and Meth.

[35] L. G. Atencio, J. F. Amann, R. L. Boudrie and C. L. Morris, Nucl. Inst. and Meth. 187 (1981) 381. 
[36] C. L. Morris, Nucl. Inst. and Meth. 196 (1982) 263.

[37] R. D. Ransome, Ph. D. Thesis, LA-8919-T (LAMPF, Los Alamos, 1981).

[38] R. D. Ransome, S. J. Greene, C. L. Hollas, B. E. Bonner, M. W. McNaughton, C. L. Morris and H. A. Thiessen, Nucl. Inst. and Meth. 201 (1982) 309.

[39] A. H. Walenta, Nucl. Inst. and Meth. 151 (1978) 461.

[40] G. A. Erskine, Nucl. Inst. and Meth. 198 (1982) 325.

[41] D. Brown and E. Sandler, Drift Chamber Electronics, LAMPF E-Division Semiannual Report LA-8069-PR (1979) 63.

[42] S. Ritt, Data Acquisition PIBETA Note, accessible at URL http://helena.

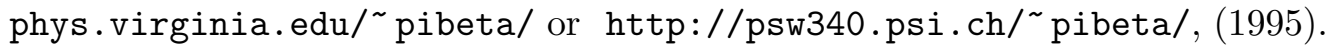

[43] F. James and M. Roos, MINUIT-Function Minimization and Error Analysis, CERNLIB D506 (CERN, Geneva, 1989).

[44] M. W. McNaughton, Introduction to the LAMPF Recoil Polarimeter (Janus) MP-13/MWM/X87-01 (LAMPF, Los Alamos, 1987).

[45] I. Supek, Ph. D. Thesis, (LAMPF, Los Alamos, 1989) unpublished.

[46] R. Brun, O. Couet, C. Vandoni, P. Zanarini and M. Goossens, PAW 2.03Physics Analysis Workstation CN/Q121 (CERN, Geneva, 1993).

[47] W. R. Leo, Techniques for Nuclear and Particle Physics Experiments, (SpringerVerlag, New York, 1987).

[48] P. J. Huber, Robust Statistics (Wiley, New York, 1981).

[49] W. H. Press, B. P. Fannery, S. A. Teukolsky and W. T. Vetterling, Numerical Recipes - Art of Scientific Computing (Cambridge University Press, Cambridge, 1986).

[50] LeCroy 1995 Research Instrumentation Catalogue (Chestnut Ridge, NY, 1995).

[51] R. Brun, F. Bruyant, M. Maire, A. C. McPherson and P. Zanarini, GEAnT 3.21 DD/EE/94-1 (CERN, Geneva, 1994).

[52] B. Rossi, Rev. Mod. Phys. 20 (1948) 537.

[53] B. K. Wright, Program OPTICS (University of Virginia, Charlottesville, 1992), unpublished.

[54] B. K. Wright, Program TkOptics (University of Virginia, Charlottesville, 1994), unpublished.

[55] J. K. Ousterhout, Tck and Tk Toolkit (Addison-Wesley, Melno Park, 1994).

[56] E. Frlež, Measurement of Bulk CsI Index of Refraction and Attenuation Length (University of Virginia, Charlottesville, 1989), unpublished. 
[57] E. Frlež, S. Ritt, K. A. Assamagan, Ch. Brönnimann, T. Flügel, B. Krause, D. Počanić, D. Renker, P. L. Slocum, N. Soić, I. Supek, ThreeDimensional Reconstruction of Light Collection Probability Function for Pure CsI Scintillators, to be submitted to Nucl. Phys. and Meth.

[58] The PIBETA Decay Experiment Home Page, accessible at URL http://helena.phys.virginia.edu/ pibeta/ or http://psw340.psi.ch/ pibeta/, (1997).

[59] The PIBETA Collaboration, Status Update of PSI Experiment R-89.01.1 (Paul Scherrer Institute, Villigen, 1995) unpublished.

[60] E. Frlež, Photonuclear Reactions in GEANT Code, accessible at URL http://helena.phys.virginia.edu/ pibeta/ or http://psw340.psi.ch/ pibeta/, (1995).

[61] J. Ahrens, H. Borchert, K. H. Czock, H. B. Eppler, H. Gimm, H. Gundrun, M. Kröning, P. Riehn, G. Sita Ram, A. Zieger and B. Ziegler, Nucl. Phys. A251 (1975) 478.

[62] A. Lepretre, H. Beil, R. Bergère, P. Carlos, J. Fagot, A De Miniac and A. Veyssière, Nucl. Phys. A367 (1981) 237.

[63] H. Hebach, A. Wortberg, and M. Gari, Nucl. Phys. A267 (1976) 425.

[64] B. L. Berman, R. L. Bramblett, J. T. Caldwell, H. S. Davis, M. A. Kelly and S. C. Fultz, Phys. Rev. 177 (1969) 1745.

[65] R. L. Bramblett, J. T. Caldwell, B. L. Berman, R. R. Harvet and S. C. Fultz, Phys. Rev. 148 (1966) 1198.

[66] G. G. Jonsson and B. Forkman, Nucl. Phys. A107 (1968) 52.

[67] S. G. Brown and S. A. Bludman, Phys. Rev. 136 (1964) 1160.

[68] E. Frlež, K. A. Assamagan, Ch. Brönnimann, T. Flügel, J. E. Koglin, B. Krause, D. W. Lawrence, R. C. Minehart, D. Počanić, D. Renker, B. G. Ritchie, S. Ritt, P. L. Slocum, and I. Supek, The Response of the PIBETA CsI Calorimeter Array to 10-70 MeV Positrons, Electrons and Tagged Photons, to be submitted to Nucl. Phys. and Meth. 
Fig. 1. The PIBETA CsI shower calorimeter consists of 240 tapered hexagonal, pentagonal and trapezial pyramids. The crystal ball has an opening for beam entry and a symmetric one for light readout from inner detectors (not shown).

Fig. 2. The dimensioned drawing of the HEX-A (top) and HEX-D1 (bottom) CsI crystal shapes.

Fig. 3. The fast-to-total light component ratio for the all studied CsI crystals. The light outputs from optically polished and unwrapped crystals were measured immediately after the crystals were received from a manufacturer with a digital oscilloscope at the average ambient temperature of $22^{\circ} \mathrm{C}$.

Fig. 4. The Monte Carlo simulation of penetrating cosmic muons interacting with the experimental apparatus. Six CsI crystals were defined inside a dark box and deposited energies and track lengths of cosmic muons and generated secondary particles in detectors were digitized.

Fig. 5. The schematic diagram of the electronics logic for the tomography apparatus. The calibration event was triggered by two-fold scintillator coincidence $\mathrm{S} 1 \times \mathrm{S} 2$. The trigger used during cosmic muon data acquisition required three-fold coincidence between two scintillators and at least one CsI detector, $(\mathrm{S} 1 \times \mathrm{S} 2) \times \sum \mathrm{CsI}_{i}$.

Fig. 6. One-dimensional residuals defined as differences between the best straight line fits from the reconstructed coordinates in the hit chambers 1 and 2 and the measured positions in the top chamber 3. The FWHM of the peak is $0.9 \mathrm{~mm}$ for $x$ coordinate and $1.5 \mathrm{~mm}$ for $y$ coordinate, respectively. The $1.2 \mathrm{~cm}$ wide plateaus contains $\sim 5 \%$ events with incorrect left-right identification and/or the events undergoing the large angle scattering in the apparatus.

Fig. 7. The Monte Carlo distribution of scattering angles of the cosmic muons intersecting a HEX-A CsI crystal shape. The root-mean-square of the scattering angle for the events with the reconstructed tracks is $0.66^{\circ}$. That value corresponds to the CsI pathlength uncertainty of $\sim 1 \mathrm{~mm}$.

Fig. 8. The average measured cosmic muon pathlength in CsI crystals was $\sim 6 \mathrm{~cm}$. An accurate determination of the track length requires the precise knowledge of the absolute crystal position with respect to the drift chambers. The number of muon tracks intersecting a CsI crystal volume is shown as a function of horizontal crystal offsets. Contours of the 25-level plot reveal a crystal position with an uncertainty of $\pm 0.05 \mathrm{~cm}$ (left panel, pentagonal CsI crystal: $x_{\text {off }}=12.32 \pm 0.05 \mathrm{~cm}$, $\left.z_{\text {off }}=30.50 \pm 0.05 \mathrm{~cm}\right)$. The same type of plot is shown for one hexagonal CsI crystal on the right panel. 
Fig. 9. Fast light output temperature coefficients for all analyzed CsI calorimeter detectors. The mean value is $-1.4 \% /{ }^{\circ} \mathrm{C}$, with the temperature coefficients ranging from $-4 \% /{ }^{\circ} \mathrm{C}$ to $+4 \% /{ }^{\circ} \mathrm{C}$. Temperature changes inside the light-tight box (filled markers) and average ADC values (open circles) for one detector with the negative temperature coefficient of $-1.9 /{ }^{\circ} \mathrm{C}$ are shown in the inset. Data points shown in the inset span one week of data acquisition.

Fig. 10. LED spectra in one CsI detector recorded for five different values of the LED driving voltage. The horizontal scale is a raw ADC count of the fast CsI scintillation component (a $100 \mathrm{~ns}$ ADC gate) corrected for the pedestal offset. The equivalent energy range covered is $10-100 \mathrm{MeV}$. The photoelectron statistics can be determined by measuring the width of a photodiode peak $\sigma_{E}^{2}$ as a function of a spectrum mean value $\bar{E}$. The linear dependence described by Eq. (6) is demonstrated in the inset. The inverse slope of the straight line equals the number of photoelectrons created per unit energy deposition.

Fig. 11. The number of photoelectrons per $\mathrm{MeV}$ of deposited energy for all studied CsI calorimeter modules. The left panel shows the distribution for the full-sized hexagonal and pentagonal CsI detectors viewed with three inch phototubes. The right panel is the equivalent histogram for the smaller half-hexagonal and trapezial detectors equipped with two inch phototubes.

Fig. 12. The simulated light collection probability as a function of axial $(z)$ and transverse $(x)$ coordinates for an optically ideal hexagonal detector (HEX-A) with a two-layer Teflon wrapping. The size of the histogrammed two-dimensional bin is $1 \times 1 \mathrm{~cm}^{2}$. The average light collection probability with a three inch phototube is $23 \%$.

Fig. 13. The Monte Carlo light collection probability as a two dimensional function of axial and transverse coordinates for an ideal half-hexagonal detector (HEX-D1) with a Teflon wrapping. The average light collection probability with a two inch photocathode is $12 \%$.

Fig. 14. Relative light per unit pathlength $\mathrm{ADC} / d$ as a function of axial position of energy deposition is shown for six CsI crystals. The phototubes were always positioned on the surface with the larger values of the $z$ coordinate. Plotted values were corrected for temperature variation effects and the PMT gain drifts.

Fig. 15. Detected light per unit pathlength as a function of transverse coordinates of energy deposition shown at the fixed distance along the crystal axis $\left(z_{0}=6 \pm 1 \mathrm{~cm}\right)$ for six CsI detectors.

Fig. 16. Detected light per unit pathlength as a function of transverse position of energy deposition shown at the fixed axial distance $\left(z_{0}=18 \pm 1 \mathrm{~cm}\right)$ for six CsI crystals. 
Fig. 17. The light nonuniformity coefficients $a_{z 1}$ and $a_{z 2}(\% / \mathrm{cm})$, determined separately for front $(z \leq 10 \mathrm{~cm})$ and back $(z \geq 10 \mathrm{~cm})$ crystal sections, are shown here in a scatter-plot for 74 CsI detectors. The full-sized hexagonal and pentagonal detectors are represented with the full marker points, while the open circles indicate the half-hexagonal and trapezial detector shapes. The dotted line is the result of a "robust" straight-line fit: $a_{z 1}(\% / \mathrm{cm}, z \leq 10 \mathrm{~cm})=-1.44+1.19 \cdot a_{z 2}(\% / \mathrm{cm}, z \geq 10 \mathrm{~cm})$.

Fig. 18. The $662 \mathrm{keV}{ }^{137} \mathrm{Cs}$ gamma source and the background spectrum measured at the source axial position $z=10 \mathrm{~cm}$ from the front face of the pentagonal S003 crystal.

Fig. 19. The comparison of normalized light output (ADC reading/pathlength) along the axis of three different CsI crystals deduced from the cosmic muon tomography data (full line), and as measured with the ${ }^{137} \mathrm{Cs}$ gamma source scan (dotted line).

Fig. 20. The predicted fractional energy resolution $\mathrm{FWHM}_{(220)} / E_{(220)}$ of the PIBETA calorimeter for positrons and photons in the energy range $10-120 \mathrm{MeV}$. The GEANT-deduced relative $\mathrm{FWHM}_{(220)}$ for the sum over 220 CsI ADCs is shown both for an ideal homogeneous detector (full lines) as well as for a nonuniform calorimeter (dotted lines). Notice that the zero value on the vertical scale is suppressed. The curves represent the parameterizations quoted in the text.

Fig. 21. The GEANT-deduced $\mathrm{FWHM}_{(\mathrm{NN})} / E_{(\mathrm{NN})}$ for the sum over nearest-neighbor CsI ADCs is shown both for an ideal homogeneous detector as well as for a nonuniform calorimeter.

Fig. 22. Nonlinearity of the PIBETA calorimeter energy response for positrons and photons in the energy range $10-120 \mathrm{MeV}$. The ratio of the detected ADC peak position to the incident particle energy is calculated in a GEANT simulation. Results for an ideal homogeneous detector and a nonuniform calorimeter are shown separately. The normalization is given by $\mathrm{ADC} / E\left(70 \mathrm{MeV} e^{+}, \gamma\right)=1$.

Fig. 23. Nonlinearity of the PIBETA calorimeter energy response for positrons and photons in the energy range $10-120 \mathrm{MeV}$. Ratio of the detected peak position of summed nearest-neighbor ADC spectra to the incident particle energy is calculated in a GEANT simulation. 
Fig. 24. The predicted PIBETA calorimeter spectra of the monoenergetic $69.8 \mathrm{MeV}$ positrons and $70.8 \mathrm{MeV}$ photons. The $220 \mathrm{ADC}$ values above the $1.0 \mathrm{MeV}$ TDC threshold were summed. A GEANT simulation assumed different numbers of photoelectrons/MeV and linear axial light collection nonuniformities as extracted in the tomography analysis. The results for the "realistic" detector depend on the treatment of the CsI crystal surfaces and the details of the gain matching algorithm.

Fig. 25. The predicted PIBETA calorimeter spectra of the monoenergetic $69.8 \mathrm{MeV}$ positrons and $70.8 \mathrm{MeV}$ photons. The ADC value for the CsI crystal with the maximum ADC value and the ADC readings of its nearest neighbors were summed. A GEANT simulation assumed different numbers of photoelectrons/MeV and linear axial light collection nonuniformities for each detector, as measured in the tomography apparatus. 
Table 1

Average scintillation properties of hexagonal and pentagonal PIBETA CsI calorimeter shapes PENTAs, HEX-As, HEX-Bs, HEX-Cs and HEX-Ds (59 crystals). All crystals were polished and wrapped in two layers of a Teflon membrane and one layer of aluminized Mylar film. The light yields are normalized to the temperature of $18^{\circ} \mathrm{C}$. All other parameters were measured at the average laboratory room temperature of $22^{\circ} \mathrm{C}$.

\begin{tabular}{cc} 
Bicron CsI & Kharkov CsI \\
Crystals (22) & Crystals (37) \\
\hline
\end{tabular}

\begin{tabular}{lcc}
\hline \hline Fast-to-Total Ratio $(100 \mathrm{~ns} / 1 \mu \mathrm{s}$ gate $)$ & 0.835 & 0.739 \\
\# Photoelectrons/MeV $(100 \mathrm{~ns}$ ADC gate $)$ & 83.4 & 65.1 \\
\# Photoelectrons/MeV $(1 \mu \mathrm{s}$ ADC gate $)$ & 99.9 & 88.0 \\
Fast Light Temp. Coefficient $\left(\% /{ }^{\circ} \mathrm{C}\right)$ & -1.20 & -1.61 \\
Total Light Temp. Coefficient $\left(\% /{ }^{\circ} \mathrm{C}\right)$ & -1.25 & -1.40 \\
Axial Nonuniformity Coefficient $(\% / \mathrm{cm})$, & & \\
$\quad z \leq 10 \mathrm{~cm}, 100 \mathrm{~ns}$ ADC gate & -0.200 & -0.124 \\
Axial Nonuniformity Coefficient $(\% / \mathrm{cm})$, & & -1.61 \\
$\quad z \geq 10 \mathrm{~cm}, 100 \mathrm{~ns}$ ADC gate & -1.62 & \\
Transverse Nonuniformity Coefficient $(\% / \mathrm{cm})$, & & -0.500 \\
$\quad z_{0}=6 \mathrm{~cm}, 100 \mathrm{~ns}$ ADC gate & -0.400 & -1.20 \\
Transverse Nonuniformity Coefficient $(\% / \mathrm{cm})$, & \\
$\quad z_{0}=18 \mathrm{~cm}, 100 \mathrm{~ns}$ ADC gate & -1.10 & \\
\hline
\end{tabular}


Table 2

Average scintillation properties of half-hexagonal and trapezial PIBETA CsI calorimeter shapes HEX-D1/2s and VETO-1/2s (15 crystals). All crystals were polished and wrapped in two layers of Teflon foil and one layer of aluminized Mylar.

\begin{tabular}{|c|c|c|}
\hline & $\begin{array}{l}\text { Bicron CsI } \\
\text { Crystals (3) }\end{array}$ & $\begin{array}{l}\text { Kharkov CsI } \\
\text { Crystals (12) }\end{array}$ \\
\hline Fast-to-Total Ratio (100 ns/1 $\mu$ s gate) & 0.806 & 0.728 \\
\hline \# Photoelectrons/MeV (100 ns ADC gate) & 34.5 & 31.5 \\
\hline \# Photoelectrons/MeV (1 $\mu \mathrm{s}$ ADC gate $)$ & 42.8 & 43.3 \\
\hline Fast Light Temp. Coefficient $\left(\% /{ }^{\circ} \mathrm{C}\right)$ & -2.08 & -0.63 \\
\hline Total Light Temp. Coefficient $\left(\% /{ }^{\circ} \mathrm{C}\right)$ & -1.78 & -0.69 \\
\hline \multicolumn{3}{|l|}{ Axial Nonuniformity Coefficient $(\% / \mathrm{cm})$, } \\
\hline$z \leq 10 \mathrm{~cm}, 100 \mathrm{~ns}$ ADC gate & -0.326 & -0.500 \\
\hline \multicolumn{3}{|l|}{ Axial Nonuniformity Coefficient $(\% / \mathrm{cm})$, } \\
\hline$z \geq 10 \mathrm{~cm}, 100 \mathrm{~ns}$ ADC gate & -1.00 & -1.83 \\
\hline \multicolumn{3}{|l|}{ Transverse Nonuniformity Coefficient $(\% / \mathrm{cm})$, } \\
\hline$z_{0}=6 \mathrm{~cm}, 100 \mathrm{~ns}$ ADC gate & -0.755 & -0.827 \\
\hline \multicolumn{3}{|l|}{ Transverse Nonuniformity Coefficient $(\% / \mathrm{cm})$, } \\
\hline$z_{0}=18 \mathrm{~cm}, 100 \mathrm{~ns}$ ADC gate & -4.10 & -5.14 \\
\hline
\end{tabular}


Table 3

The predicted energy resolutions and tail contributions for $69.8 \mathrm{MeV} e^{+}$and $70.8 \mathrm{MeV} \gamma$ 's events in the full PIBETA calorimeter. The light output in photoelectrons $/ \mathrm{MeV}$ and the linear axial light collection nonuniformities measured for individual CsI crystals $\left(a_{z 1, z 2}=\right.$ TOMOGRAPHY) were used in a GEANT simulation. The values for the perfect optically homogeneous crystals $\left(a_{z 1, z 2}=0\right)$ were shown for comparison.

\begin{tabular}{ccccc}
\hline \multicolumn{1}{c}{ Parameter } & $69.8 \mathrm{MeV} e^{+}$ & & $70.8 \mathrm{MeV} \gamma$ & \\
No $E_{V}$ cut & $a_{z 1, z 2}=0$ & TOMOGRAPHY $a_{z 1, z 2}$ & $a_{z 1, z 2}=0$ & TOMOGRAPHY $a_{z 1, z 2}$ \\
\hline \hline Peak Position $(\mathrm{MeV})$ & $68.87 \pm 0.03$ & $68.90 \pm 0.03$ & $70.10 \pm 0.03$ & $69.97 \pm 0.03$ \\
FWHM $_{(220)}(\mathrm{MeV})$ & $3.66 \pm 0.03$ & $4.10 \pm 0.03$ & $3.98 \pm 0.03$ & $4.76 \pm 0.03$ \\
$5 \leq$ Events $\leq 54 \mathrm{MeV}(\%)$ & $6.44 \pm 0.09$ & $6.46 \pm 0.09$ & $8.20 \pm 0.10$ & $8.47 \pm 0.10$ \\
$5 \leq$ Events $\leq 55 \mathrm{MeV}(\%)$ & $6.89 \pm 0.09$ & $6.98 \pm 0.09$ & $8.86 \pm 0.10$ & $9.11 \pm 0.11$ \\
\hline$E_{V} \leq 5 \mathrm{MeV}$ & $a_{z 1, z 2}=0$ & TOMOGRAPHY $a_{z 1, z 2}$ & $a_{z 1, z 2}=0$ & TOMOGRAPHY $a_{z 1, z 2}$ \\
Peak Position $(\mathrm{MeV})$ & $68.88 \pm 0.03$ & $68.92 \pm 0.03$ & $70.07 \pm 0.03$ & $69.99 \pm 0.03$ \\
FWHM & \\
$5 \leq$ Events $\leq 54 \mathrm{MeV}(\%)$ & $1.91 \pm 0.05$ & $2.02 \pm 0.05$ & $3.92 \pm 0.07$ & $4.15 \pm 0.07$ \\
$5 \leq$ Events $\leq 55 \mathrm{MeV}(\%)$ & $2.24 \pm 0.05$ & $2.36 \pm 0.05$ & $4.46 \pm 0.07$ & $4.68 \pm 0.07$ \\
\hline
\end{tabular}


Table 4

The predicted energy resolutions and tail contributions for $69.8 \mathrm{MeV} e^{+}$and $70.8 \mathrm{MeV} \gamma$ 's events in the PIBETA clusters containing the crystal with maximum energy deposition and its nearest neighbors.

\begin{tabular}{ccccc}
\hline Parameter & $69.8 \mathrm{MeV} e^{+}$ & & & \\
No $E_{V}$ cut & $a_{z 1, z 2}=0$ & TOMOGRAPHY $a_{z 1, z 2}$ & $a_{z 1, z 2}=0$ & TOMOGRAPHY $a_{z 1, z 2}$ \\
\hline \hline Peak Position $(\mathrm{MeV})$ & $67.74 \pm 0.03$ & $67.85 \pm 0.03$ & $68.80 \pm 0.03$ & $68.65 \pm 0.03$ \\
FWHM $_{(\mathrm{NN})}(\mathrm{MeV})$ & $5.22 \pm 0.03$ & $5.90 \pm 0.03$ & $6.01 \pm 0.03$ & $6.80 \pm 0.03$ \\
$5 \leq$ Events $\leq 54 \mathrm{MeV}(\%)$ & $3.83 \pm 0.07$ & $4.01 \pm 0.07$ & $5.47 \pm 0.08$ & $5.78 \pm 0.08$ \\
$5 \leq$ Events $\leq 55 \mathrm{MeV}(\%)$ & $4.54 \pm 0.07$ & $4.73 \pm 0.08$ & $6.33 \pm 0.09$ & $6.67 \pm 0.09$ \\
\hline$E_{V} \leq 5 \mathrm{MeV}$ & $a_{z 1, z 2}=0$ & TOMOGRAPHY $a_{z 1, z 2}$ & $a_{z 1, z 2}=0$ & TOMOGRAPHY $a_{z 1, z 2}$ \\
Peak Position $_{(\mathrm{MeV})}$ & $67.75 \pm 0.03$ & $67.83 \pm 0.03$ & $68.80 \pm 0.04$ & $68.64 \pm 0.03$ \\
FWHM $_{(\mathrm{NN})}(\mathrm{MeV})$ & $5.52 \pm 0.03$ & $5.83 \pm 0.03$ & $5.92 \pm 0.03$ & $6.76 \pm 0.03$ \\
$5 \leq$ Events $\leq 54 \mathrm{MeV}(\%)$ & $3.53 \pm 0.07$ & $3.73 \pm 0.07$ & $5.28 \pm 0.08$ & $5.59 \pm 0.08$ \\
$5 \leq$ Events $\leq 55 \mathrm{MeV}(\%)$ & $4.19 \pm 0.07$ & $4.39 \pm 0.07$ & $6.10 \pm 0.09$ & $6.42 \pm 0.09$ \\
\hline
\end{tabular}




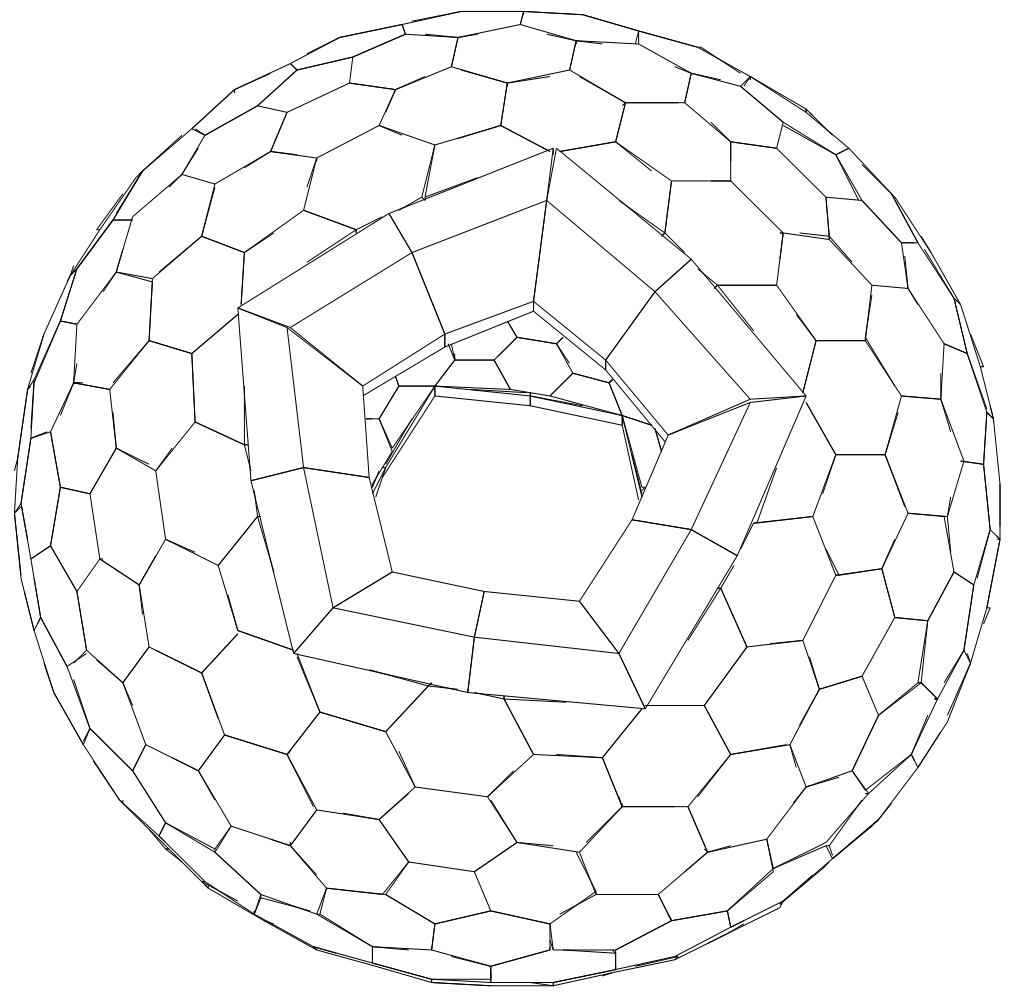

FIGURE 1 

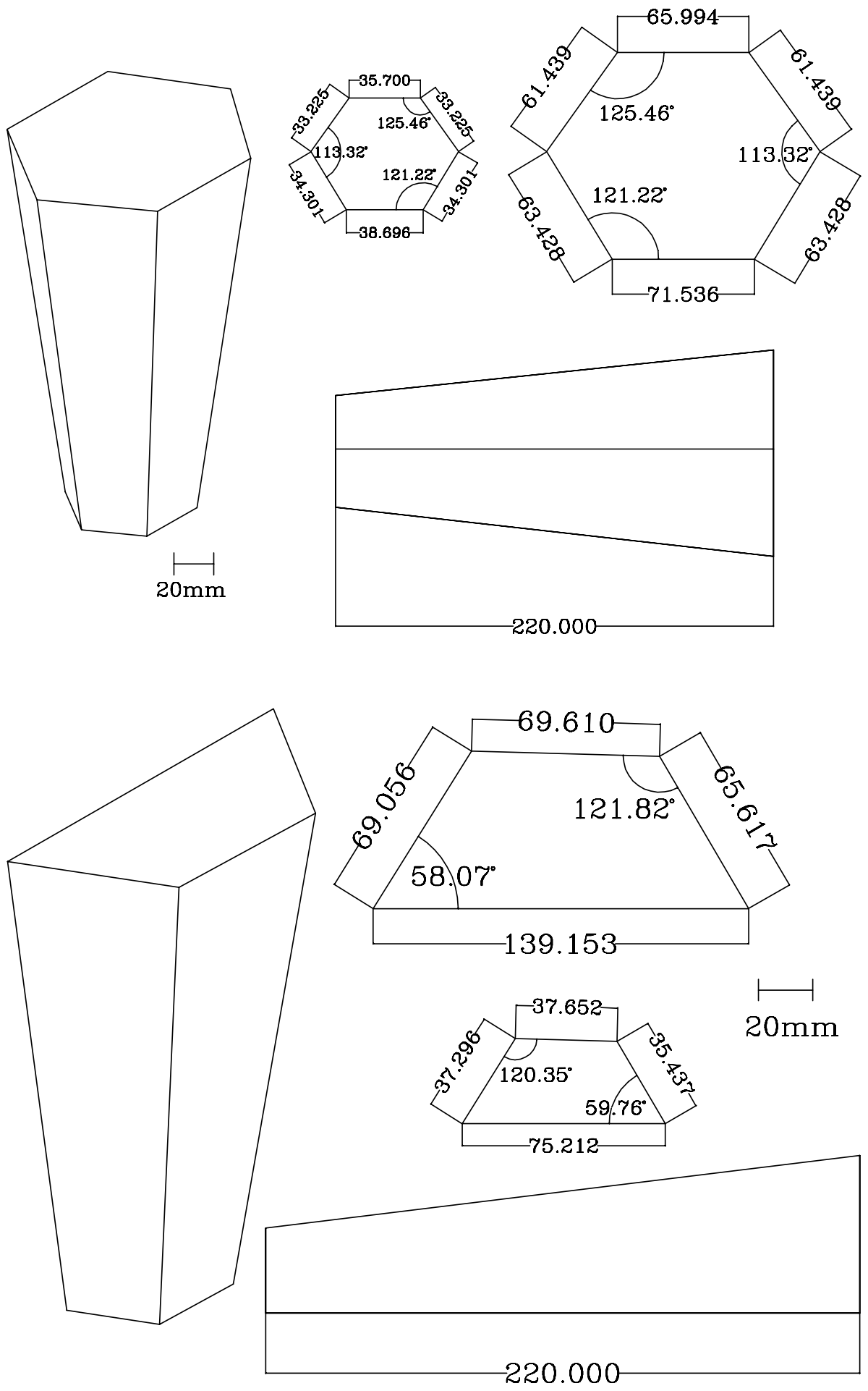

FIGURE 2 


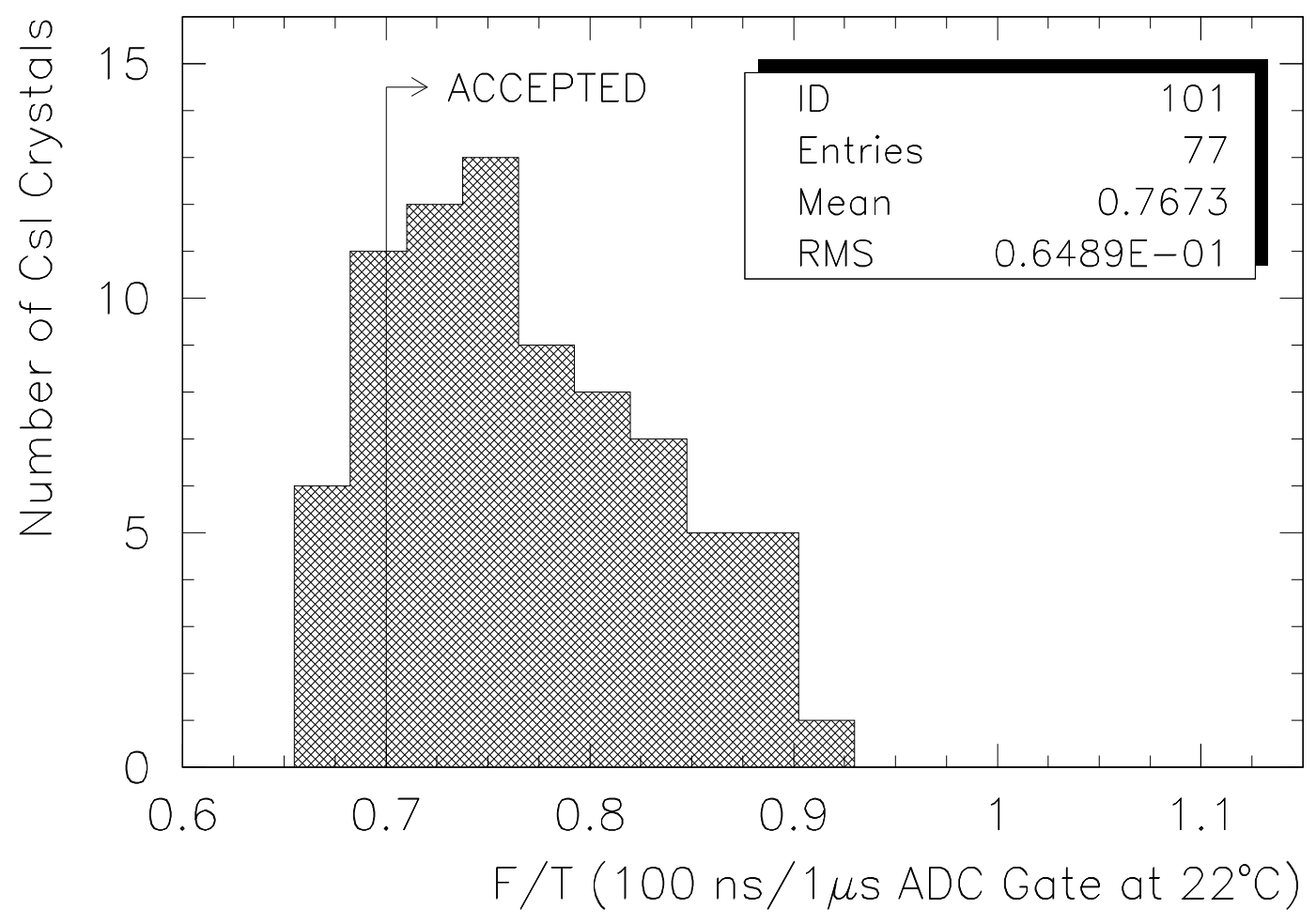

FIGURE 3 


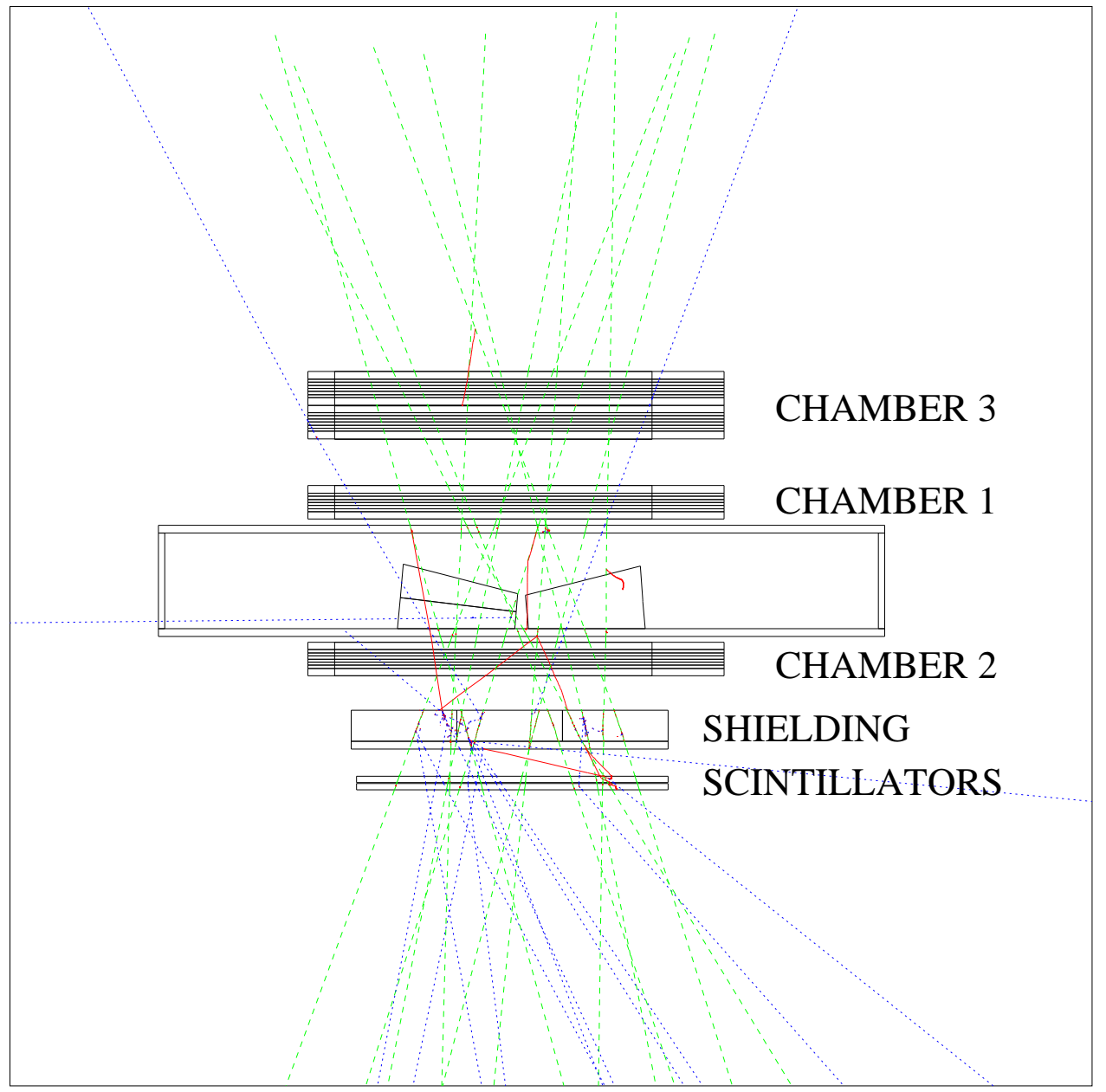

FIGURE 4 


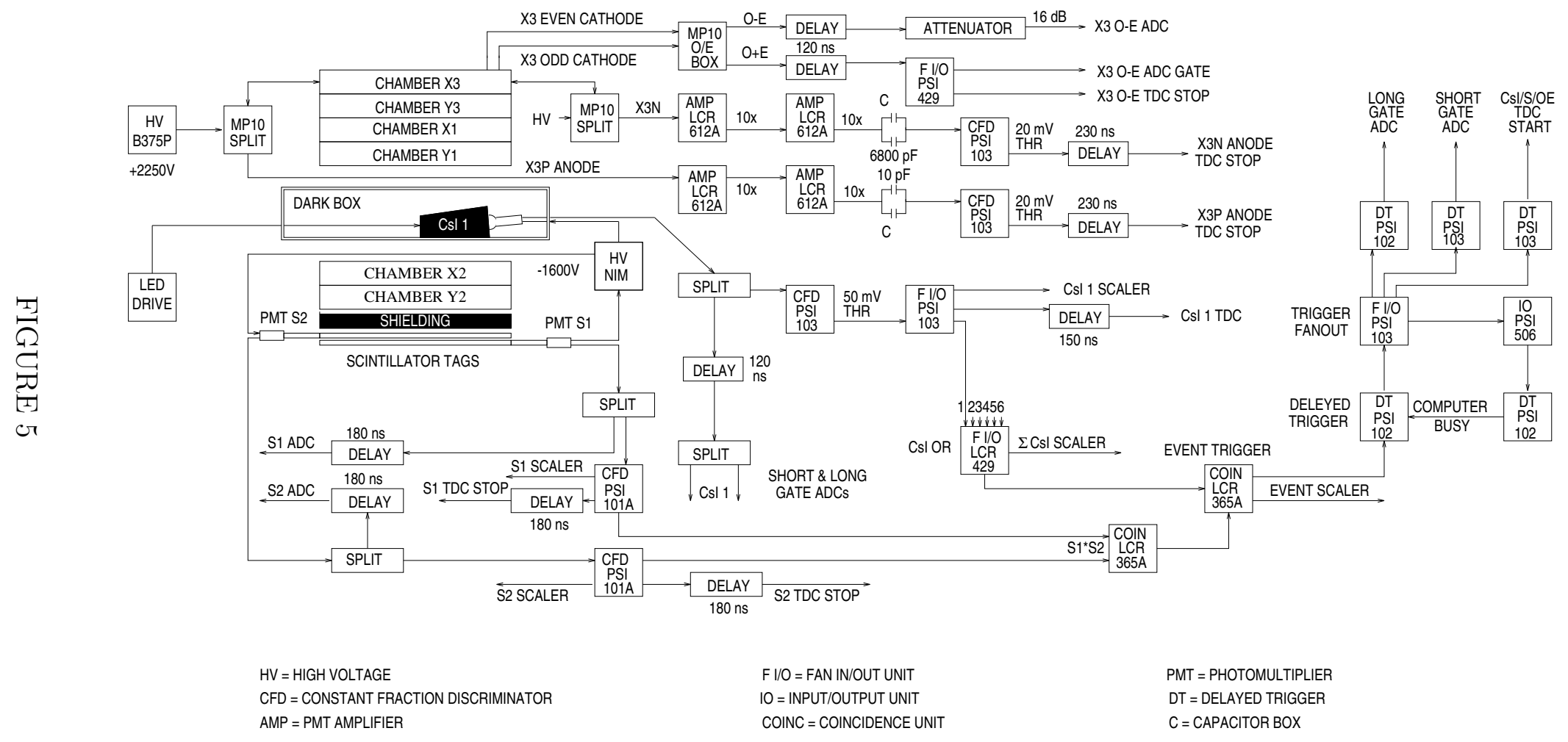



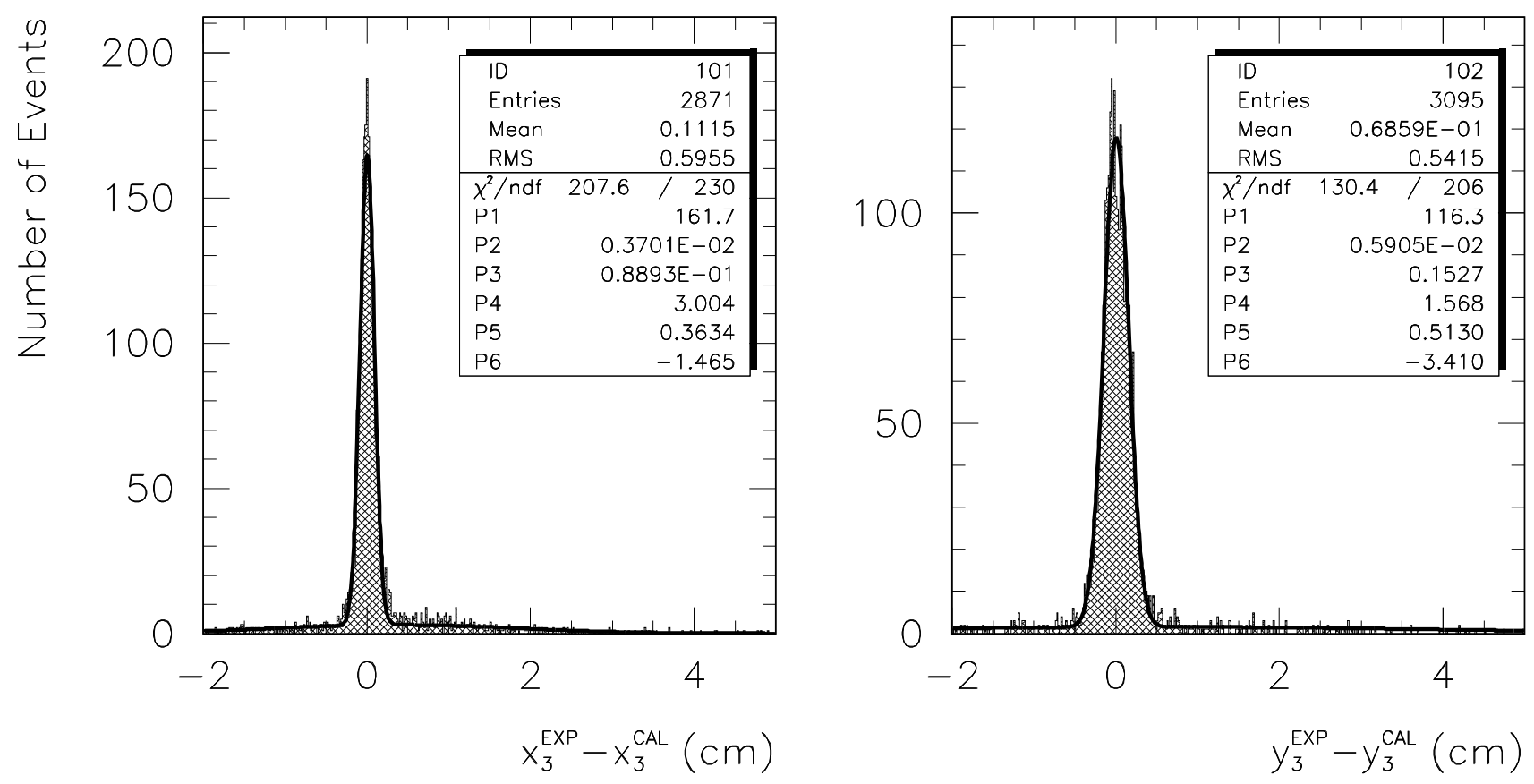

FIGURE 6 


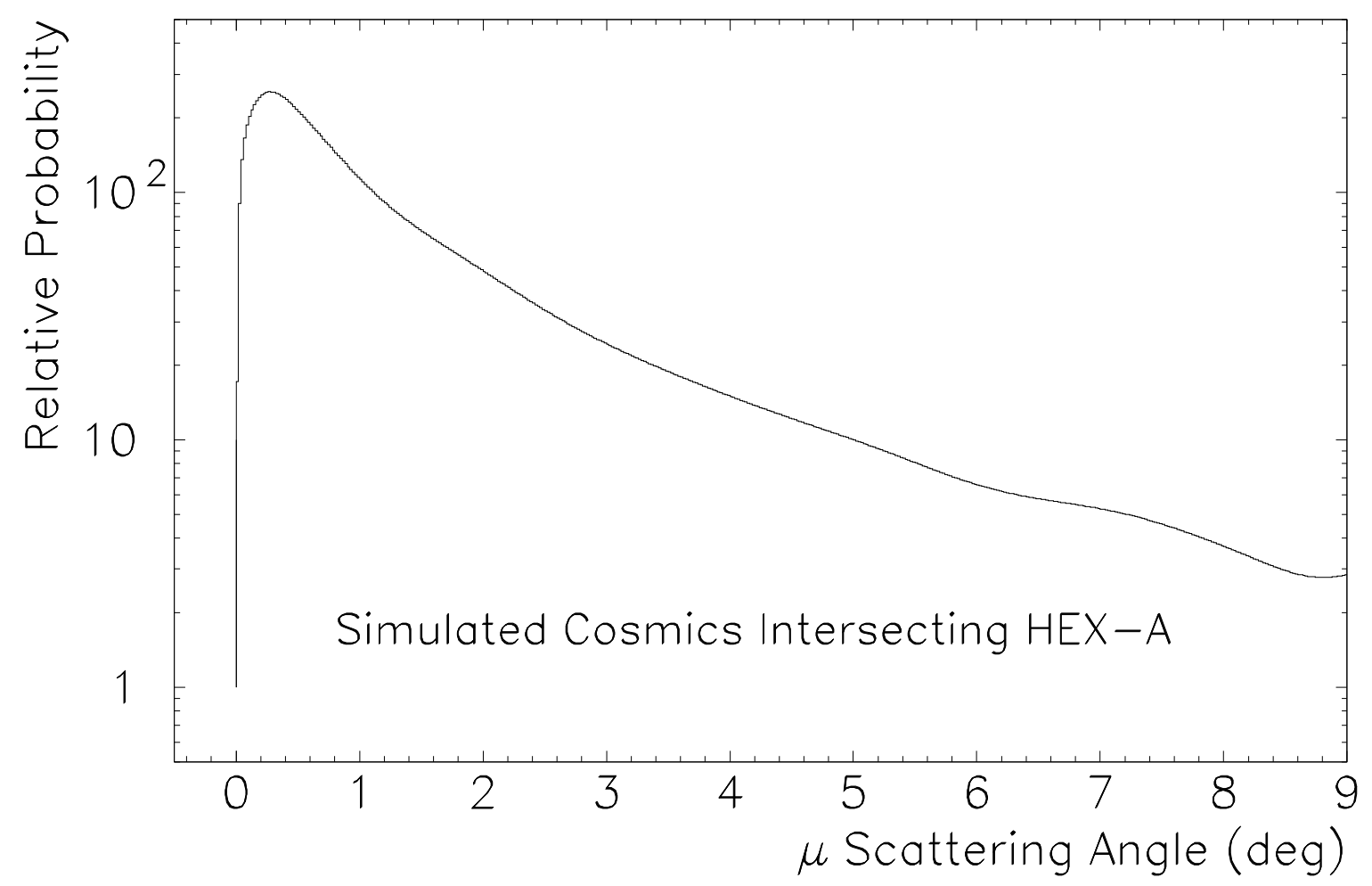

FIGURE 7 

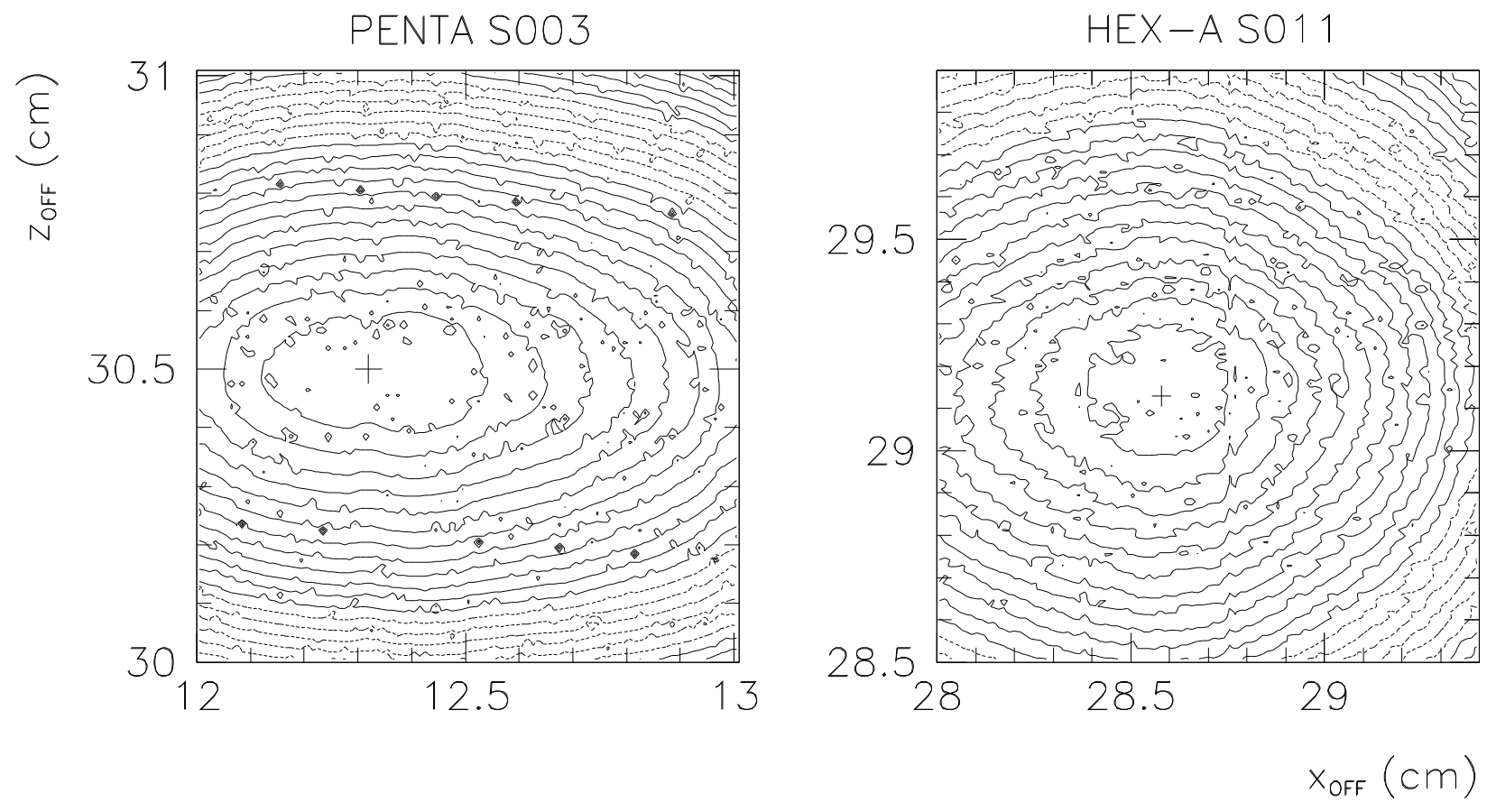

FIGURE 8 


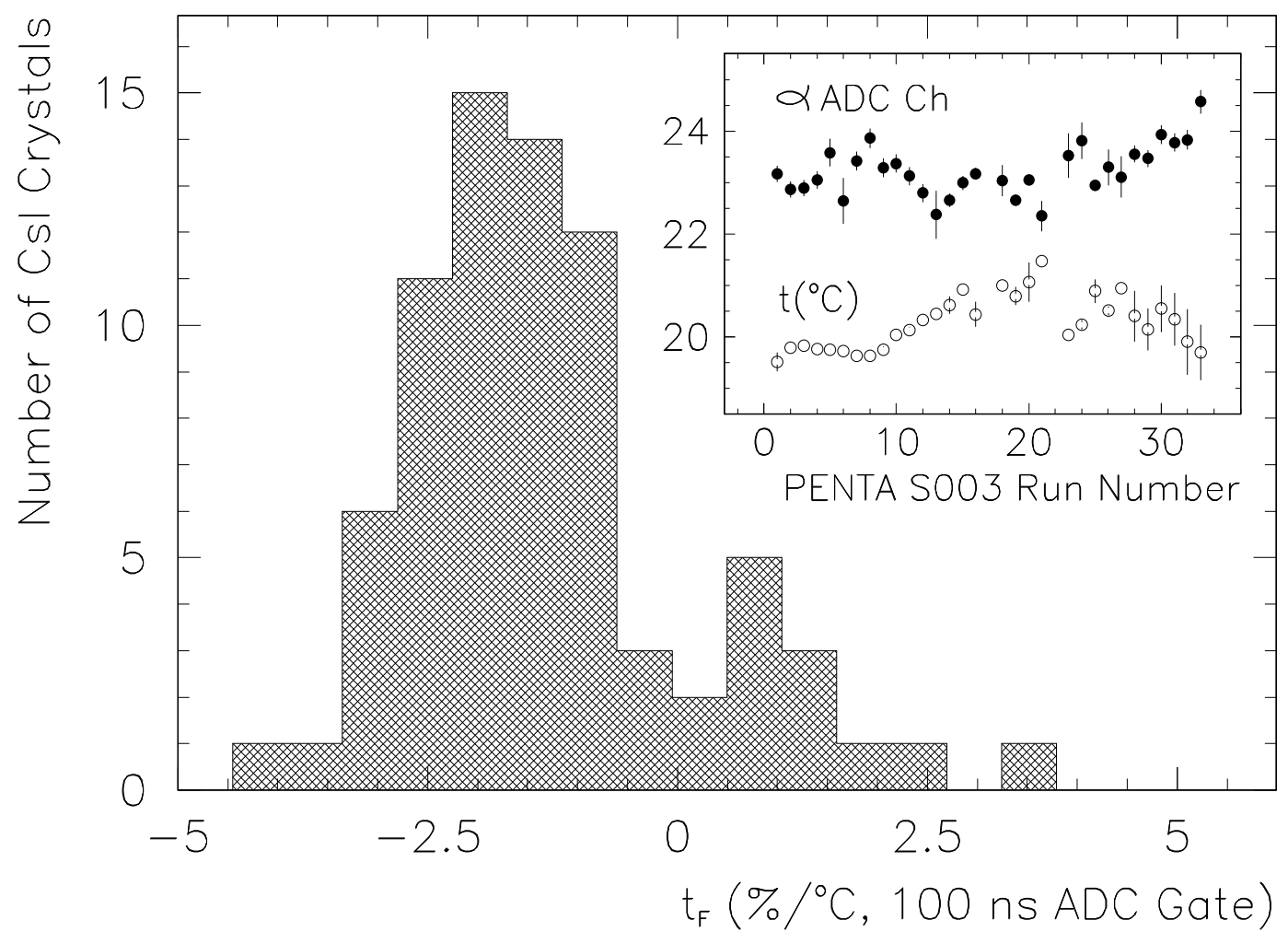

FIGURE 9 


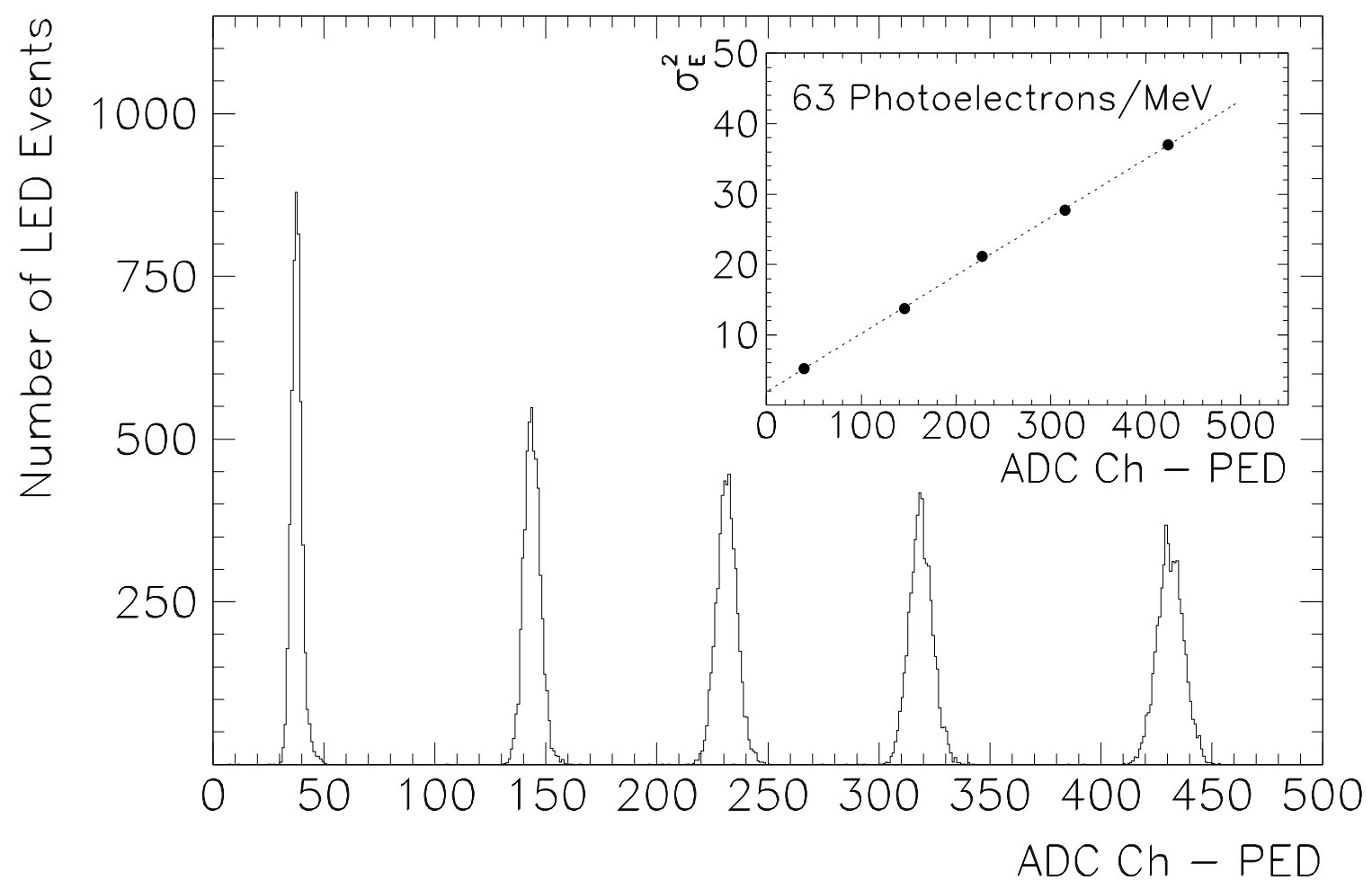

FIGURE 10 

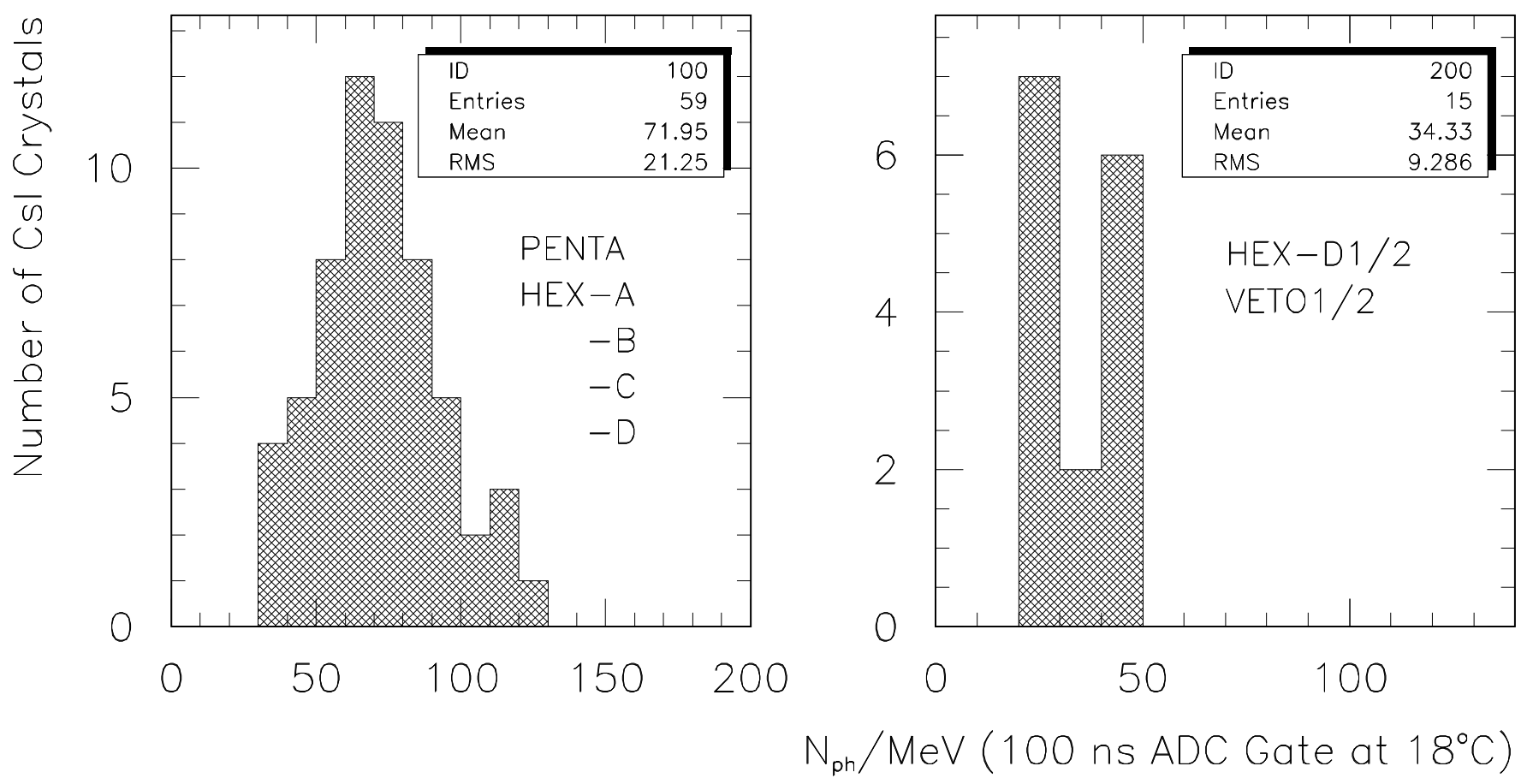

FIGURE 11 
TkOptics Simulation: HEX-A Optical Non-Uniformity

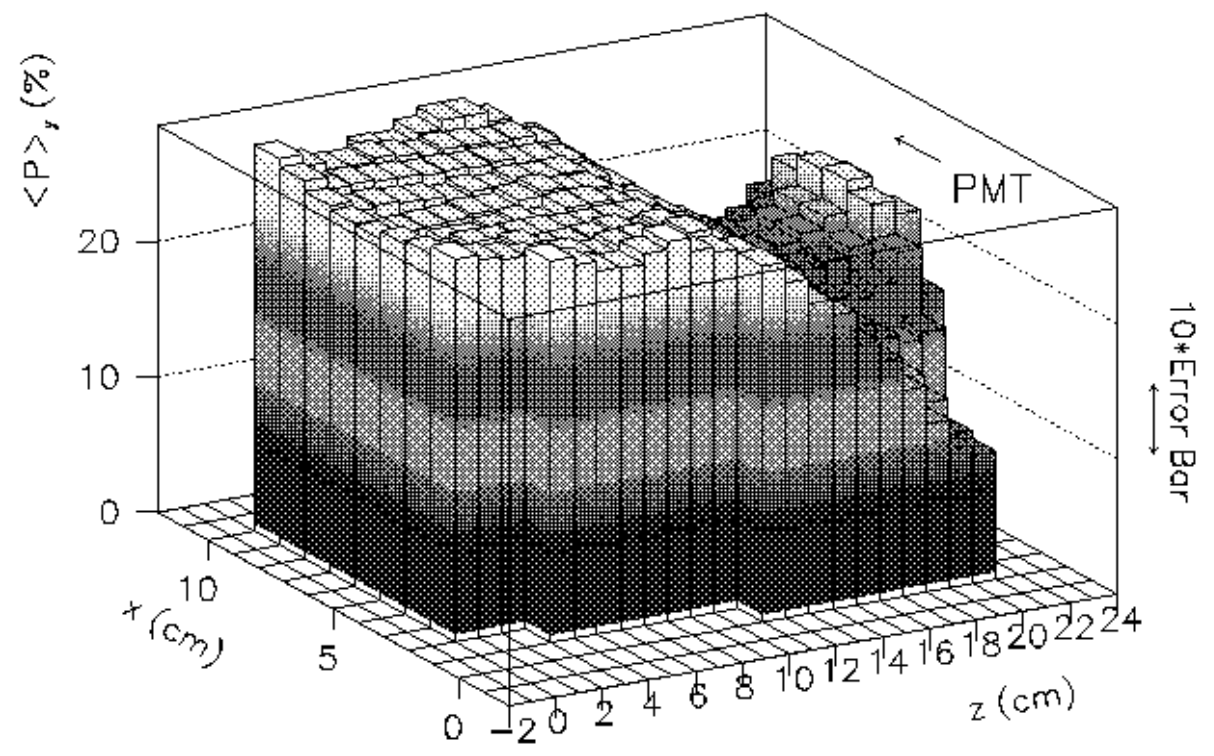

Lateral Crystal Surfaces: Specular Dielectrics $r=1.00$

Front Surface: Specular Dielectric $r=1.00$

Lateral Surface Wrapper: Diffuse Reflector $r=0.90$

Front Surface Wrapper: Diffuse Dielectric $r=0.20$

Bulk Attenuation/Scattering Length: $150 \mathrm{~cm} / 200 \mathrm{~cm}$

FIGURE 12 
TkOptics Simulation: HEX-D1 Optical Non-Uniformity

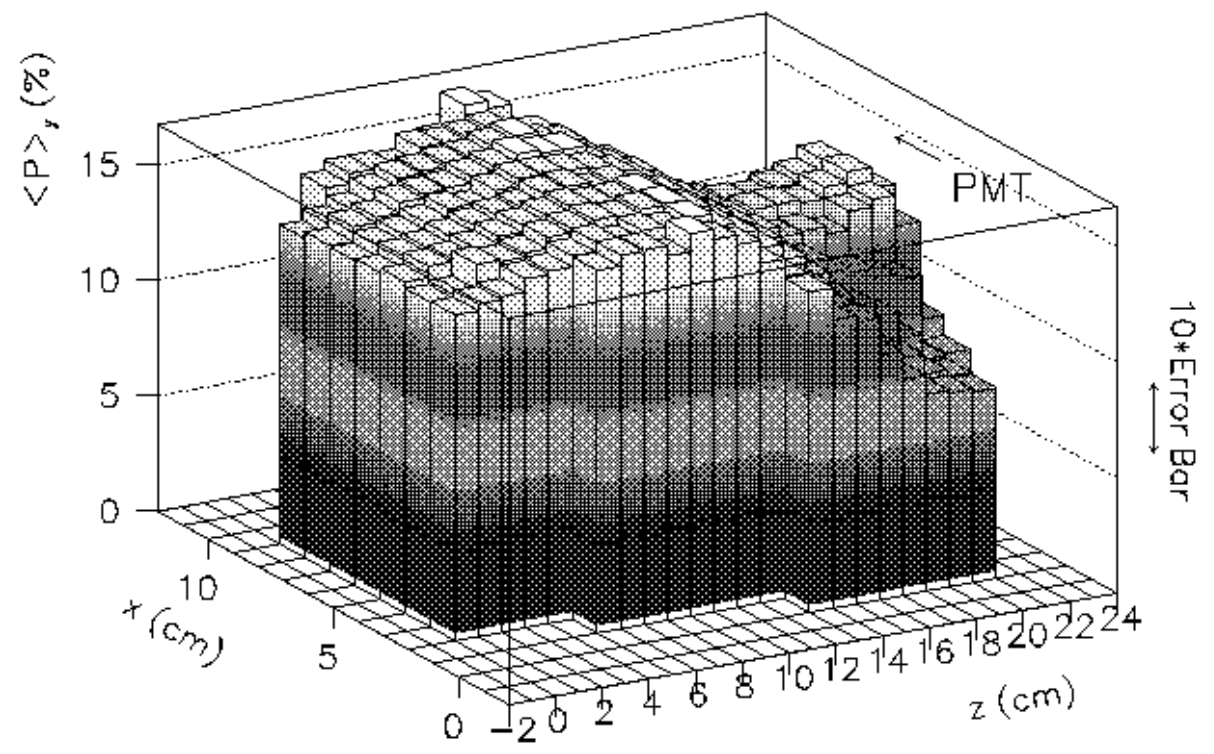

Lateral Crystal Surfaces: Specular Dielectrics $r=1.00$

Front Surface: Specular Dielectric $r=1.00$

Lateral Surface Wrapper: Diffuse Reflector $r=0.90$

Front Surface Wrapper: Diffuse Dielectric $r=0.20$

Bulk Attenuation/Scattering Length: $150 \mathrm{~cm} / 200 \mathrm{~cm}$

FIGURE 13 

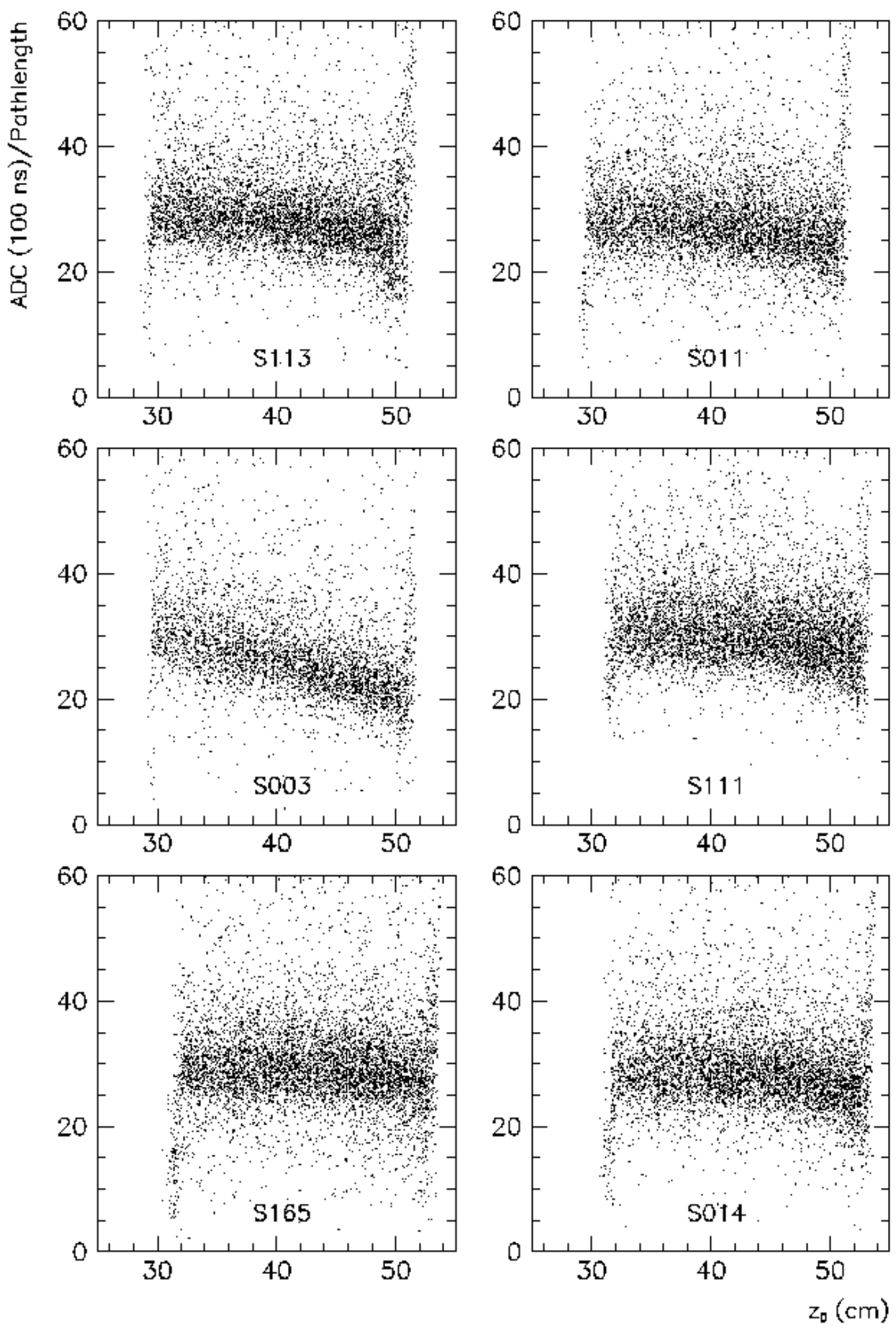

FIGURE 14 

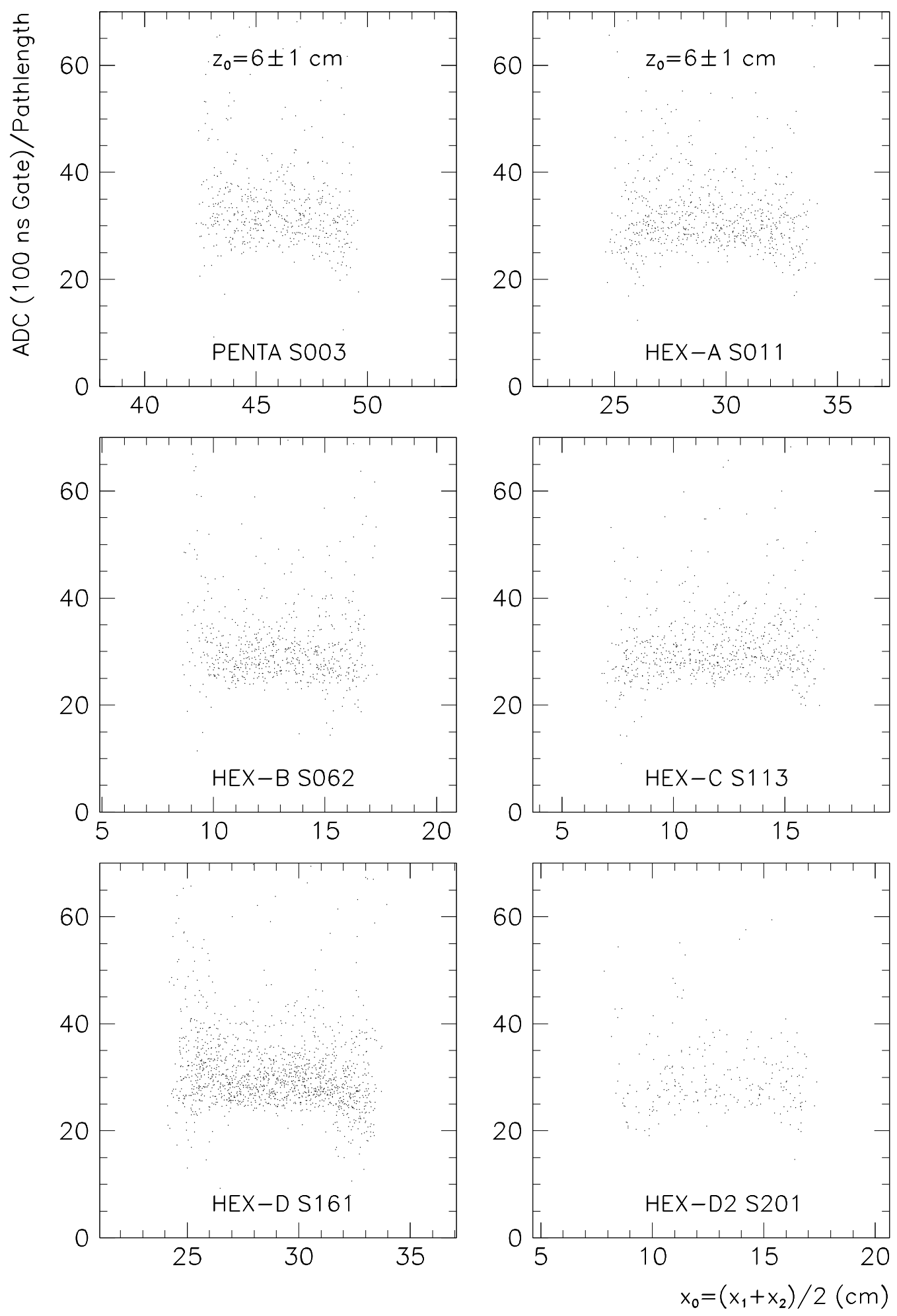

FIGURE 15 

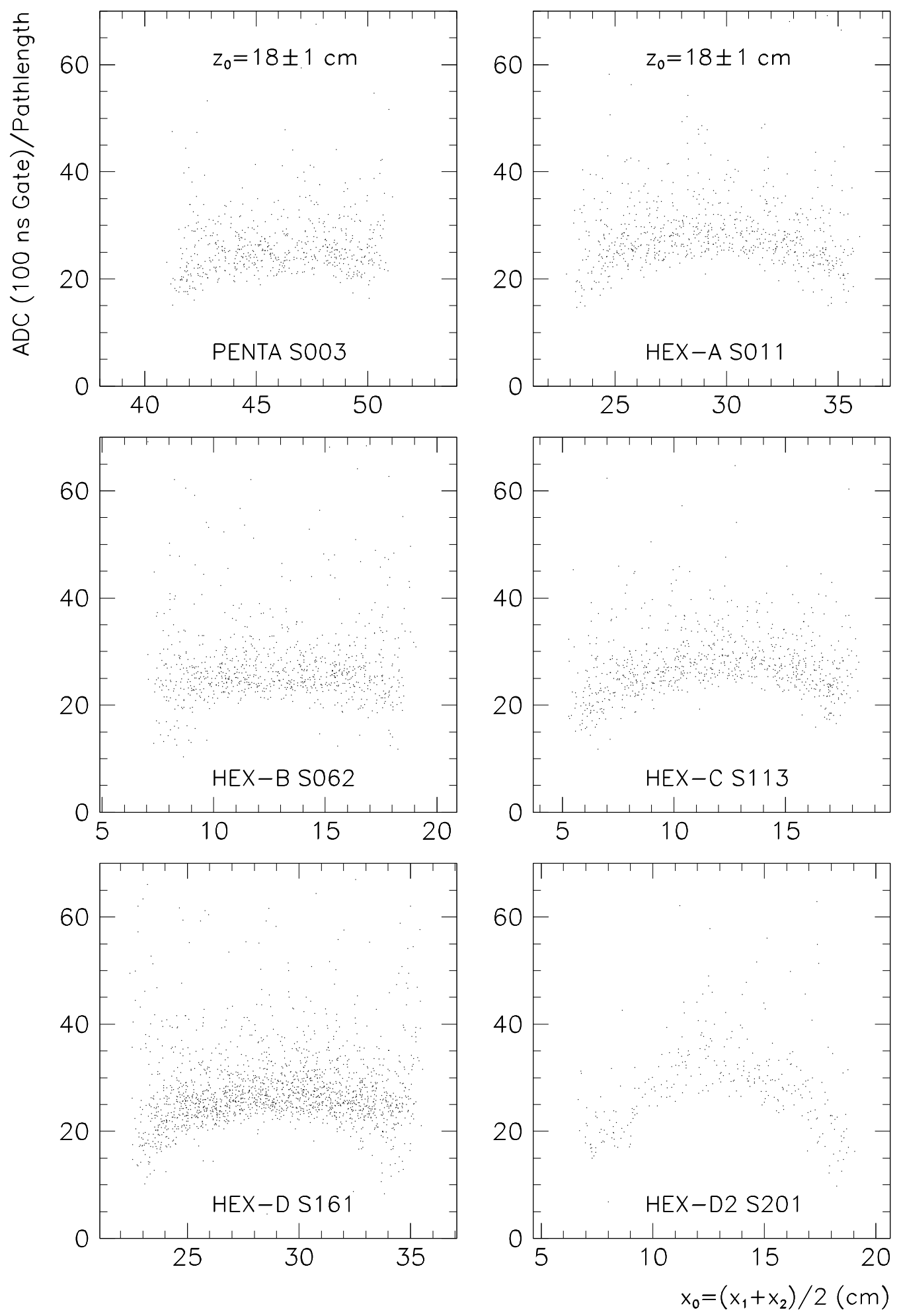

FIGURE 16 


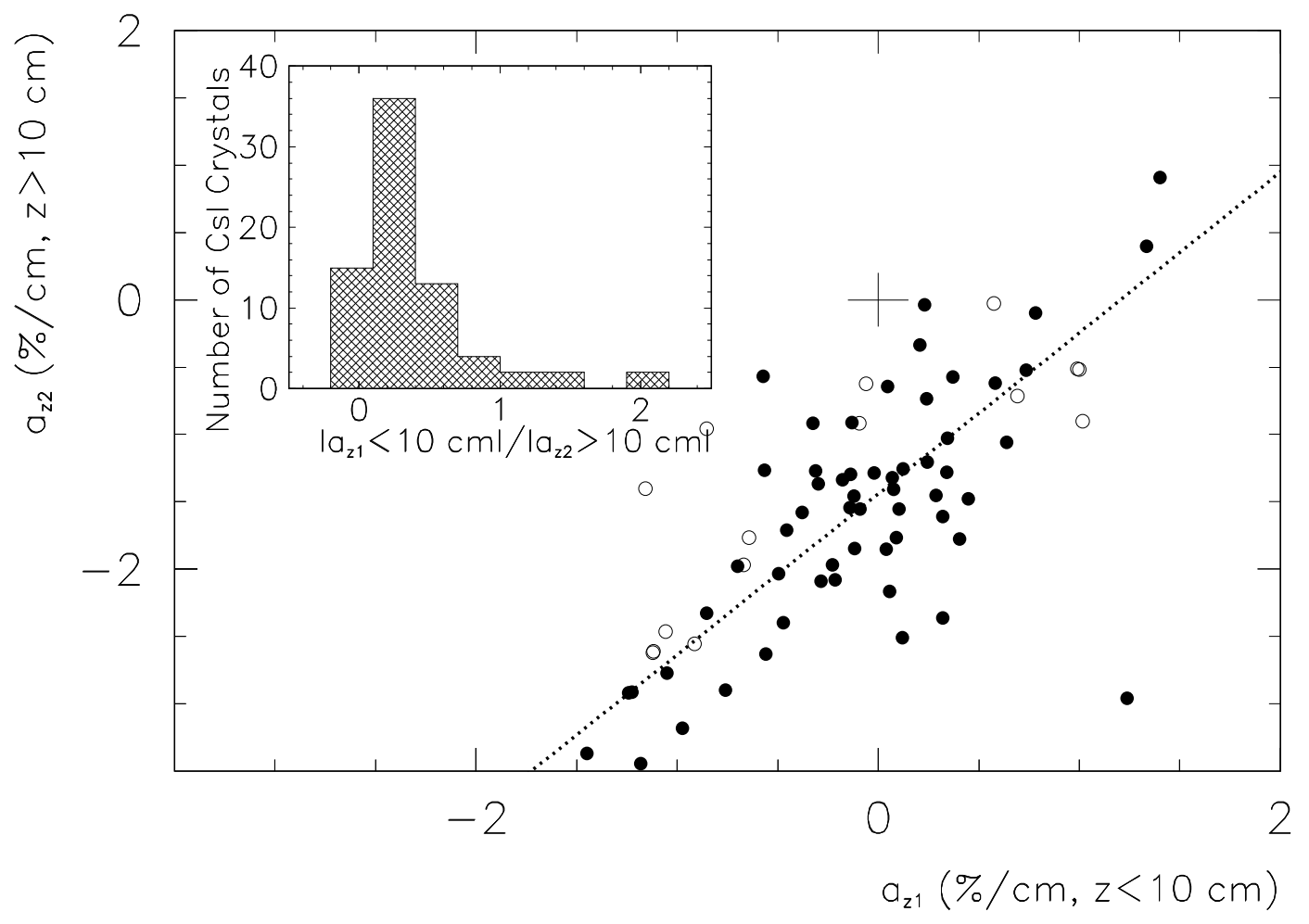

FIGURE 17 


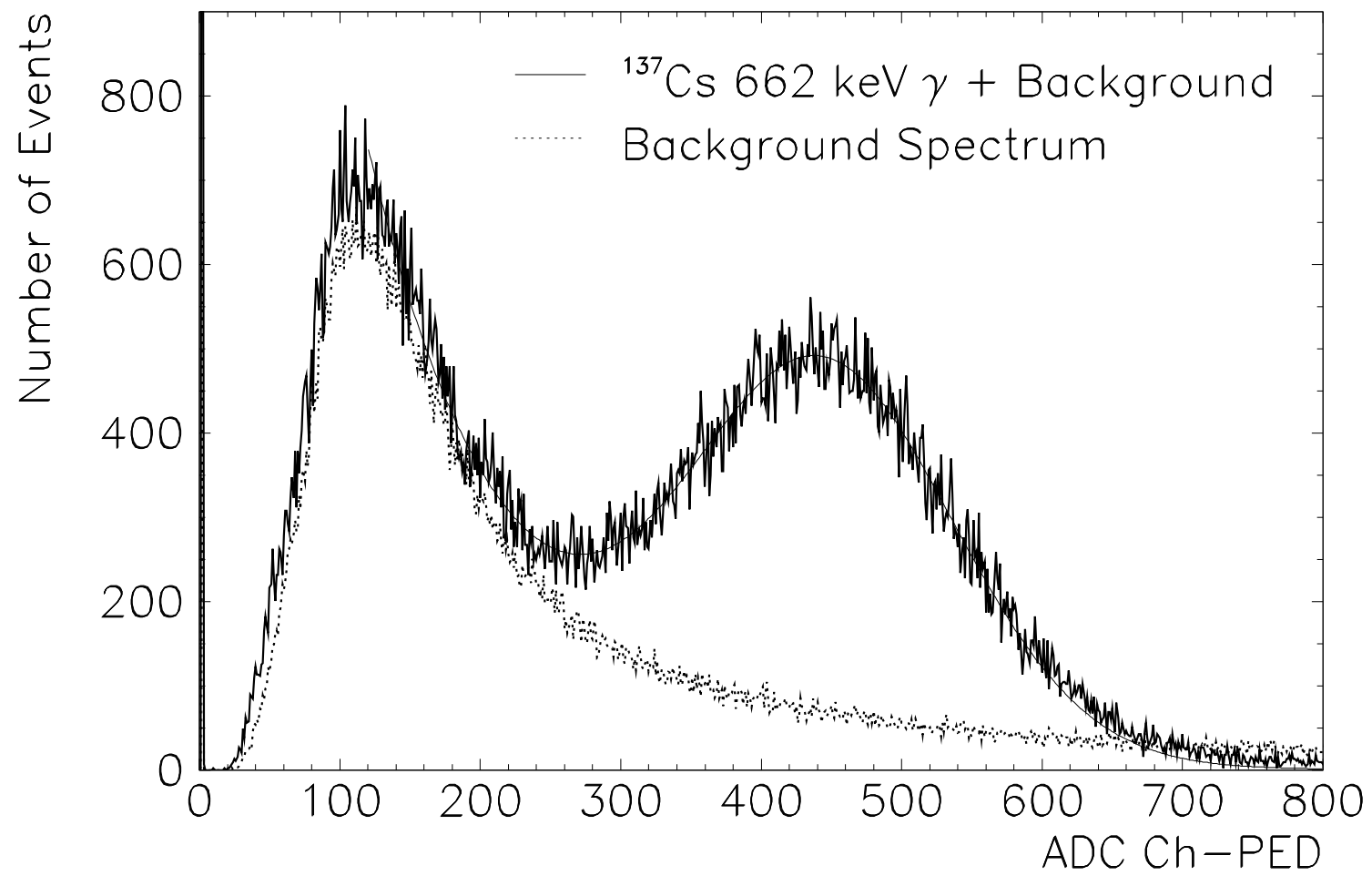

FIGURE 18 

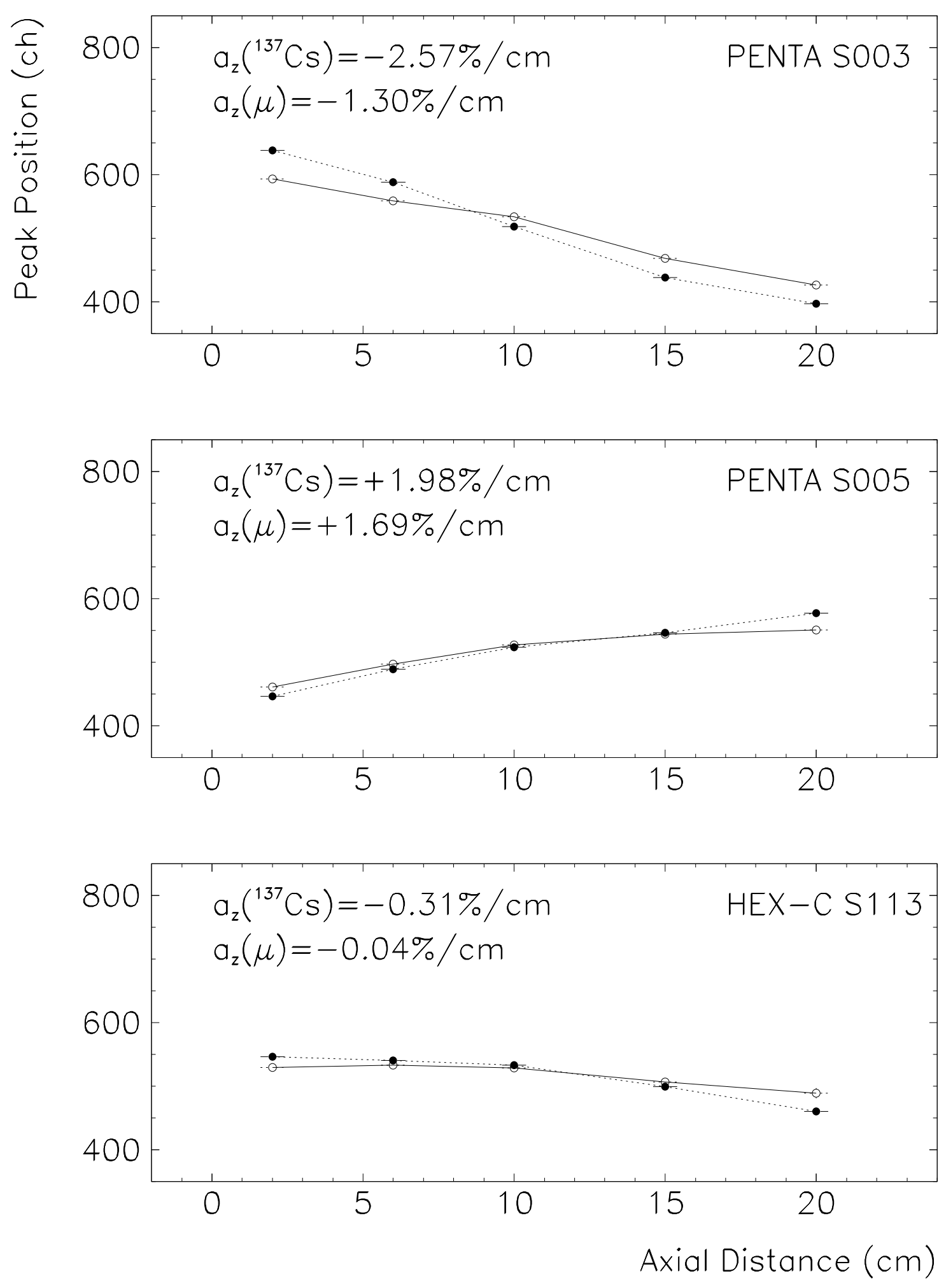

FIGURE 19 

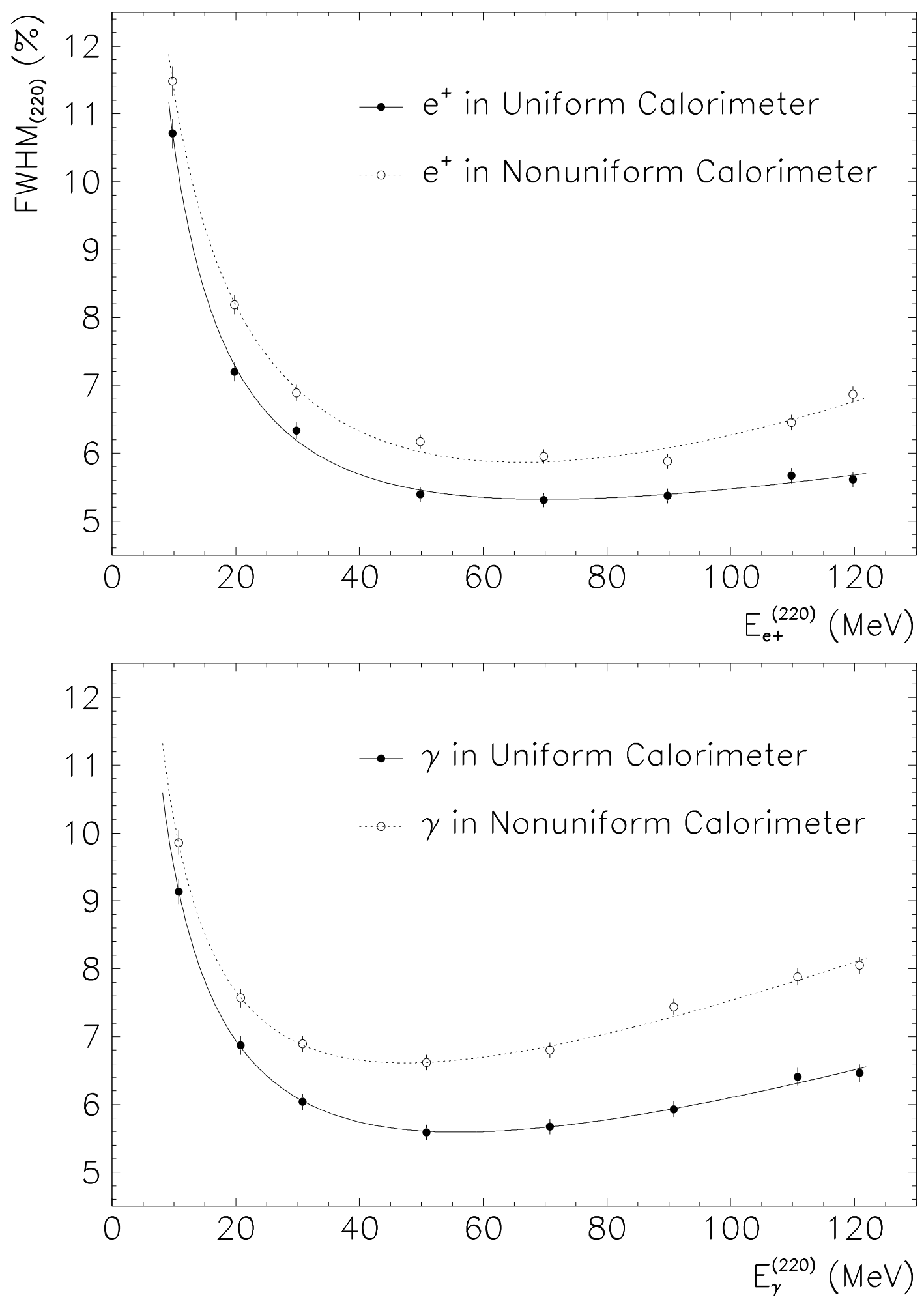

FIGURE 20 

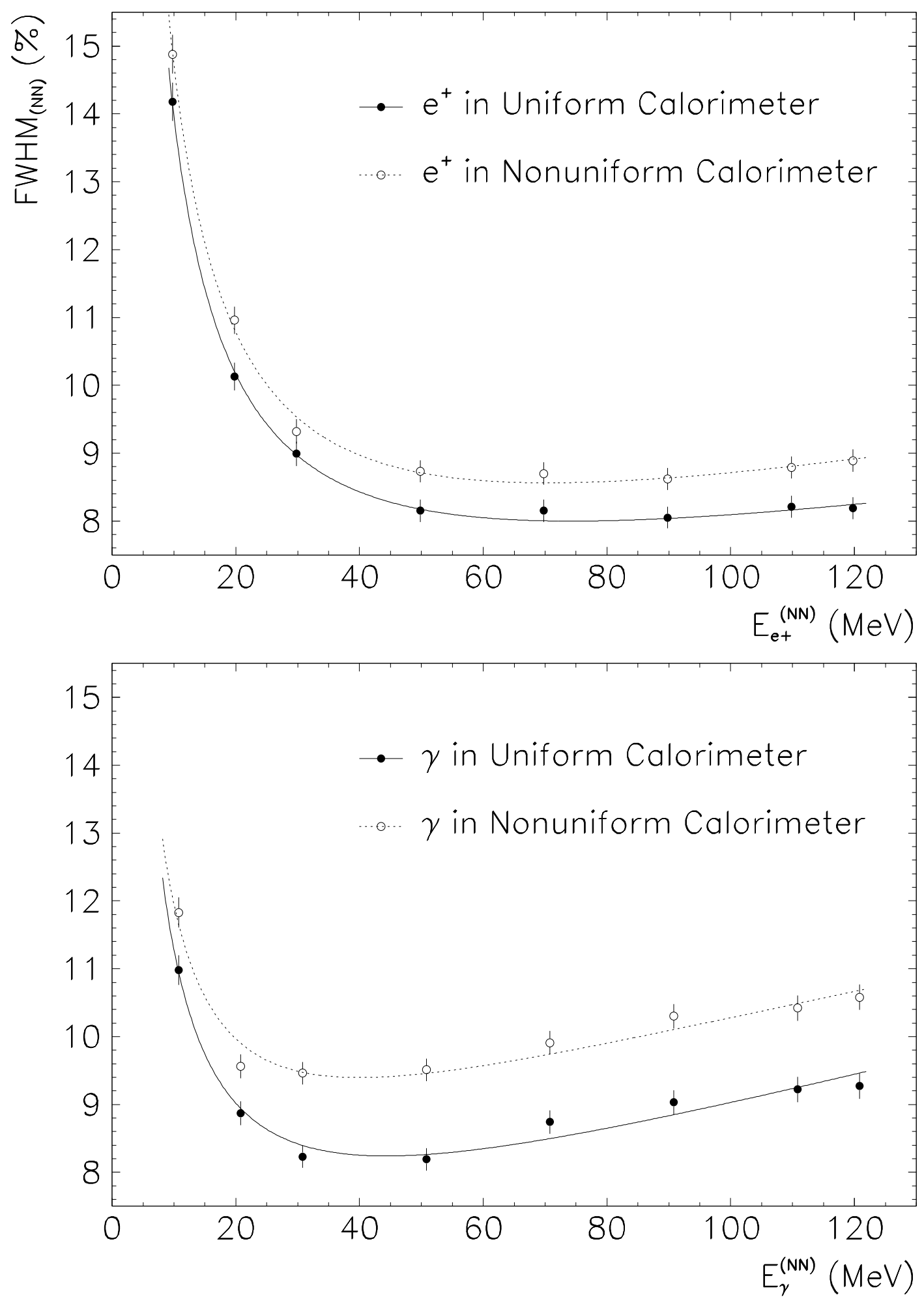

FIGURE 21 

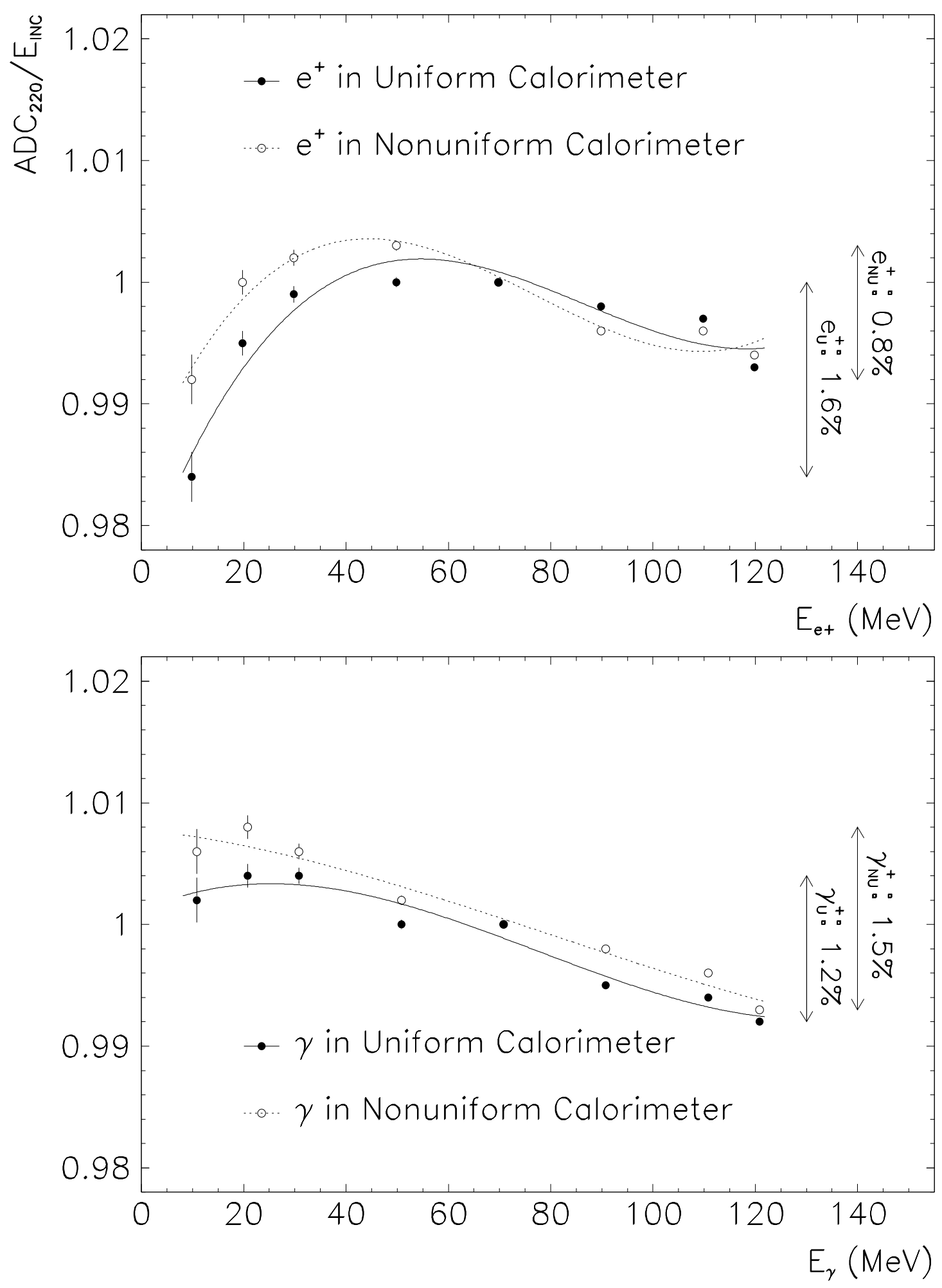

FIGURE 22 

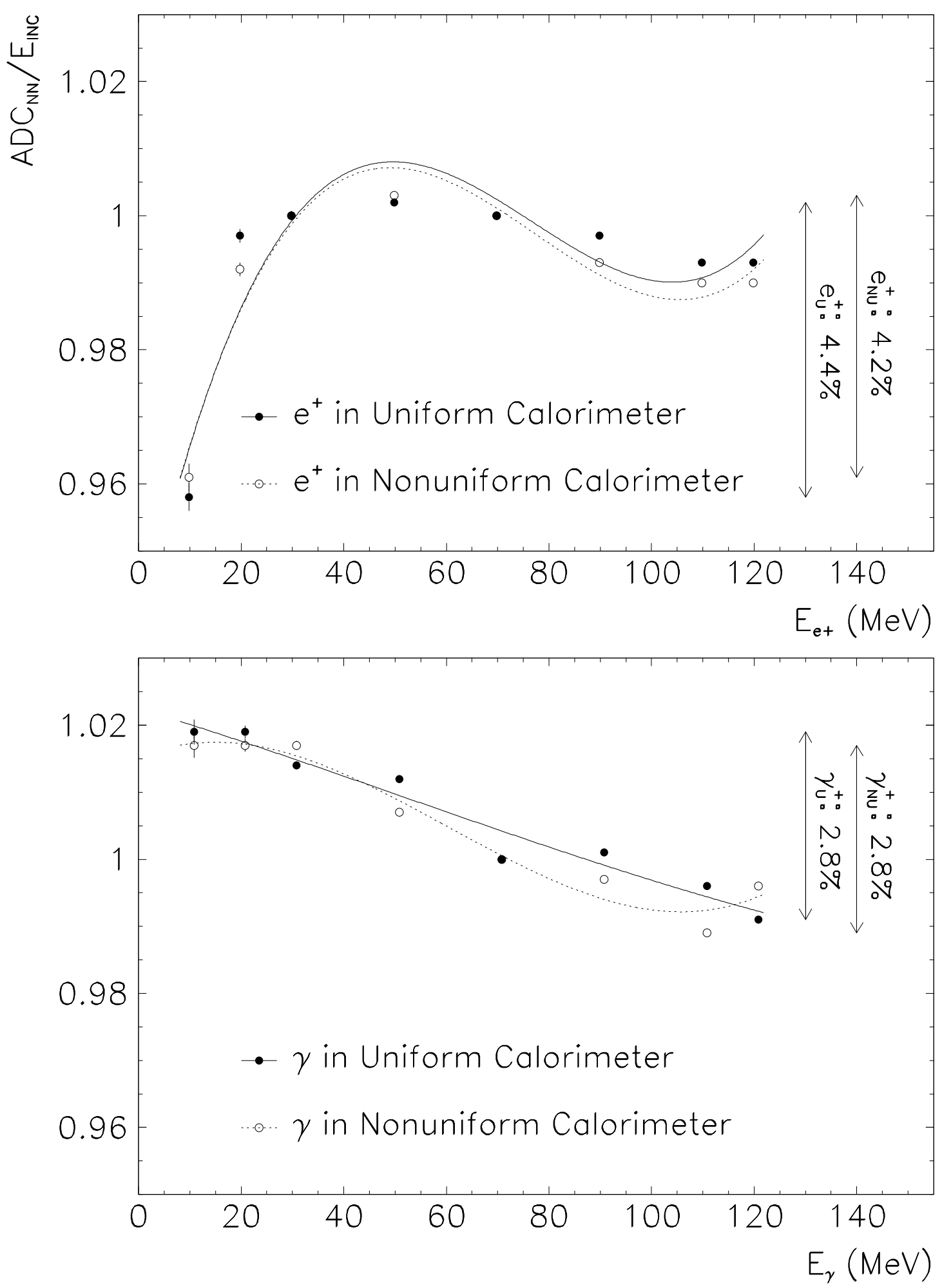

FIGURE 23 

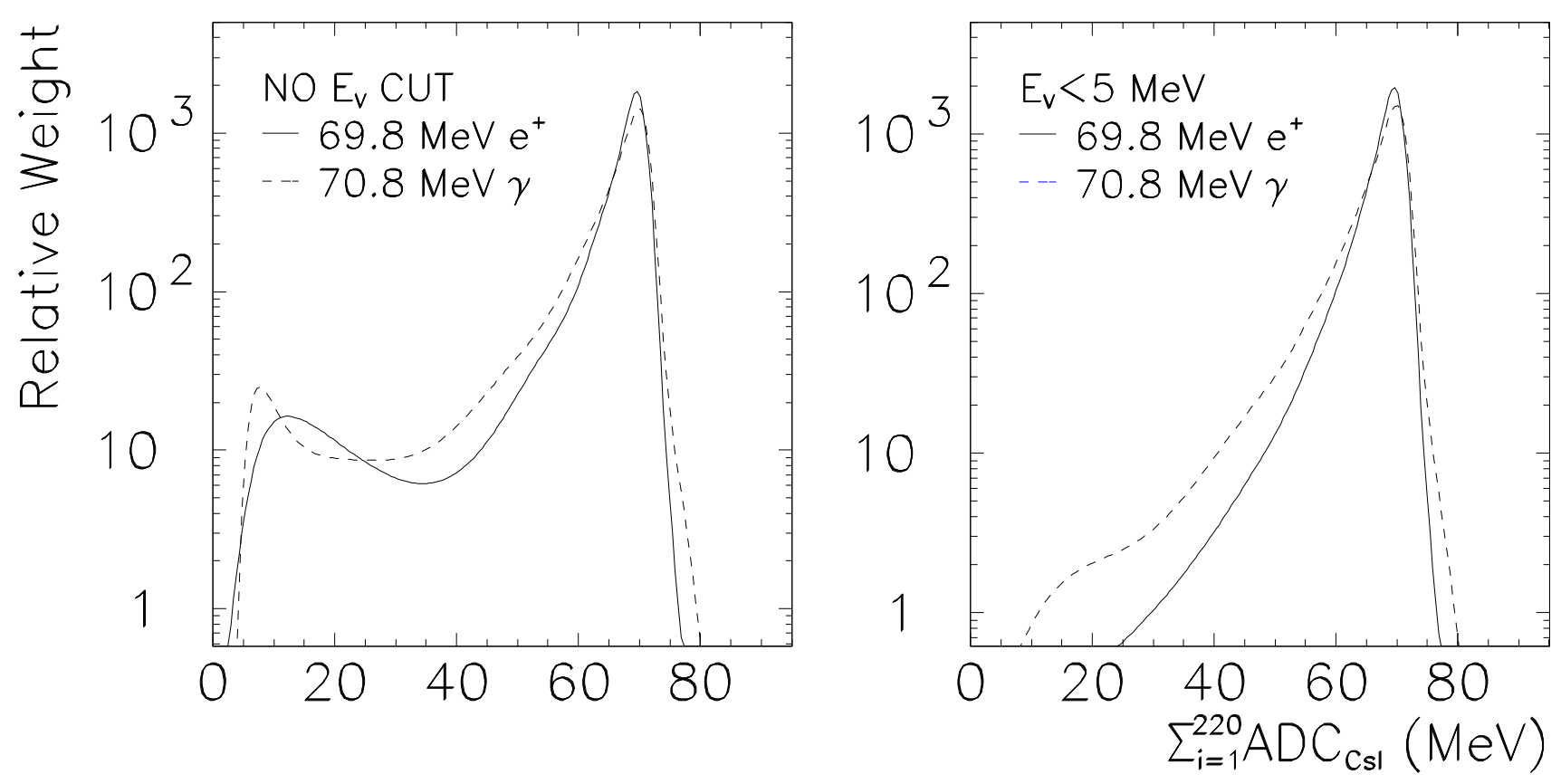

FIGURE 24 

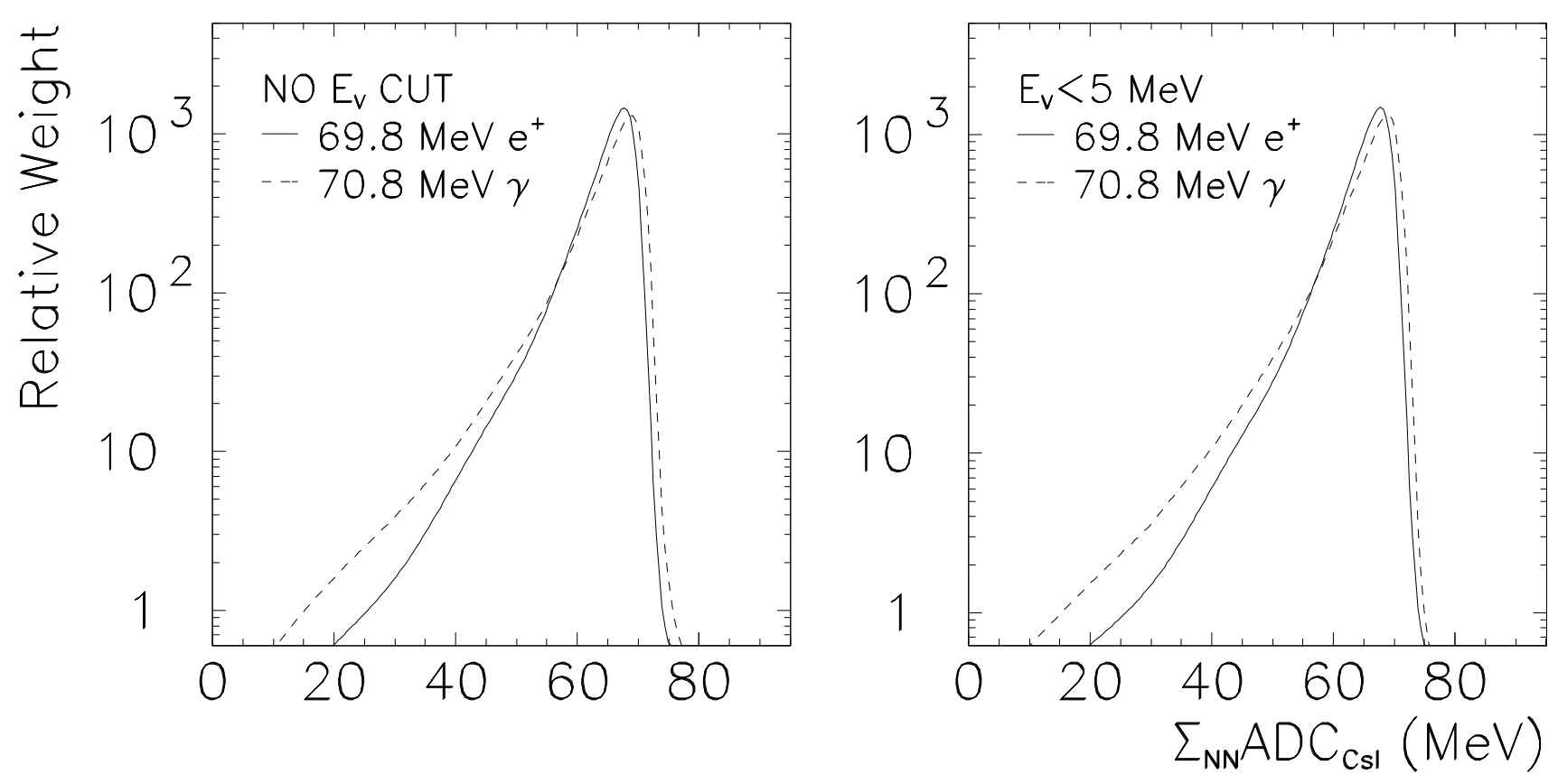

FIGURE 25 